\title{
On the Acoustic Performance of Rectangular Splitter Silencers \\ in the Presence of Mean Flow
}

\author{
Ray Kirby* \\ School of Engineering and Design, \\ Mechanical Engineering, \\ Brunel University, \\ Uxbridge, Middlesex, UB8 3PH, UK. \\ ray.kirby@brunel.ac.uk
}

\author{
Ken Amott \\ CAICE Acoustic Air Movement Ltd., \\ Riverside House, 3 Winnersh Fields, \\ Gazelle Close, Winnersh, \\ Wokingham, RG41 5QS, UK. \\ Paul T. Williams \\ School of Engineering and Design, \\ Mechanical Engineering, \\ Brunel University, \\ Uxbridge, Middlesex, UB8 3PH, UK. \\ Wenbo Duan \\ School of Engineering and Design, \\ Mechanical Engineering, \\ Brunel University, \\ Uxbridge, Middlesex, UB8 3PH, UK.
}

* Corresponding author 


\begin{abstract}
Dissipative splitter silencers are often used to reduce the noise emitted in ventilation and gas turbine systems. It is well known that the acoustic performance of a splitter silencer changes under the influence of the convective effects of a mean gas flow and so in this article a theoretical model is developed to include the effects of mean flow. The theoretical model is based on a hybrid finite element method which enables the inclusion of bull nose fairings and a perforated screen separating the mean gas flow from a bulk reacting porous material. Predictions are compared against experimental measurements obtained both with and without mean flow. Good agreement between prediction and measurement is generally observed in the absence of mean flow, although it is seen that for silencers with a low percentage open area the silencer insertion loss is over predicted at higher frequencies. When mean flow is present, problems with the experimental methodology are observed at relatively modest mean flow velocities, and so comparison between prediction and experiment is limited to relatively low face velocities. However, experiment and theory both show that the insertion loss reduces at low frequencies when mean flow is in the direction of sound propagation, and at high frequencies the influence of mean flow is generally much smaller. Following additional theoretical investigations it is concluded that the influence of mean flow on splitter silencer performance should be accounted for at low frequencies when silencer airway velocities are greater than about $20 \mathrm{~m} / \mathrm{s}$; however, at higher frequencies one may generally neglect the effect of mean flow, even at higher velocities. Predictions obtained using the hybrid method are also compared to a simplified point collocation approach and it is demonstrated that the computationally efficient point collocation method may be used to investigate the effects of mean flow in a splitter silencer without loss of accuracy.
\end{abstract}




\section{INTRODUCTION}

It is common for dissipative silencers to be used to attenuate sound radiated by fans or gas turbines. It is well known that the mean gas velocity imparts a convective effect on the sound propagation and for dissipative silencers this may significantly affect silencer performance. Integrating the effects of a mean flow field into a mathematical model suitable for predicting the acoustic performance of splitter silencers does, however, present a significant challenge. This is largely because of the size and complexity of a typical commercial dissipative silencer, as well as the complex nature of the interaction between the noise source and the (bulk reacting) absorbing material. In view of this very few articles in the literature add the effects of mean flow when analysing splitter silencer performance. Moreover, these articles are limited solely to examining the influence of mean flow on modal attenuation so that the effect of mean flow on the scattering of sound from the inlet/outlet planes of a splitter silencer has yet to be reported in the literature. This omission is, perhaps, not surprising given that a "bull-nose" fairing is normally added at either end of a silencer specifically to smooth out the mean gas flow. Therefore, in order to be consistent when including the effects of mean flow one should also incorporate the influence of the bull-nose fairing on the scattering of sound at either end of the silencer. Clearly this represents a significant computational challenge, especially for those silencers used in HVAC and gas turbine applications, which are required to perform up to an upper (octave band) frequency limit of $8 \mathrm{kHz}$. The aim of this article is to develop a mathematical model that is suitable for analysing the convective effects of mean flow on splitter silencer performance, whilst at the same time accommodating a realistic silencer design of the type typically found in HVAC and gas turbine applications. The mathematical model is validated by comparison with experimental measurements and methods for reducing computational expenditure are also explored. 
The addition of mean flow into models developed for relatively small dissipative silencers found in automotive systems is now well established [1-6]. Automotive silencers are, however, relatively straightforward to model because the absorbing material surrounds the mean gas flow so that it flows unimpeded in the central (normally circular) pipe. This is not the case for splitter silencers in which baffles of material are placed in the airway. These silencers have traditionally been investigated by assuming that they are uniform and infinite in length, which allows the eigenmodes for the silencer cross-section to be found. Relevant examples include refs. [7-12], where analytic methods have been used to derive the governing eigenequation for the silencer cross-section, which is then solved using appropriate root finding techniques. Numerical methods may also be used to solve the governing eigenequation and this approach is attractive because it avoids problems associated with analytic root finding [3]. Relevant examples include the finite element based approach of Astley and Eversman [13] for a locally reacting silencer, and Astley and Cummings [14] for a bulk reacting silencer. Moreover, the finite element method of Astley and Cummings can readily be used to obtain the eigenmodes for complex dissipative silencer designs with mean flow and in principle it is possible to use this method to examine larger splitter silencers of the type found, for example, in gas turbines. However, a modal analysis does not capture the scattering at the inlet and outlet planes of the silencer, nor does it quantify the amplitudes of the propagating eigenmodes. This is a significant limitation when attempting to predict silencer performance at higher frequencies and so this method is only of limited use for the design of splitter silencers.

The analysis of splitter silencer performance over a wide frequency range must include silencer end effects and one method of doing this is to begin by predicting silencer modal attenuation, and then to add on heuristic correction factors for each end of the silencer. Brandstätt et al. [12] used this approach for rectangular splitter silencers and, after undertaking a number of experimental measurements, arrived at correction factors for the scattering at either end of the silencers. These 
correction factors include the effects of mean flow and Brandstätt et al. show that when mean flow is in the same direction as the sound propagation the silencer insertion loss drops, whereas if the mean flow is reversed the insertion loss increases. This behaviour was also observed by Cummings and Sormaz [10], albeit for the attenuation of an individual mode. Brandstätt et al. [12] were able to obtain reasonable agreement between their semi-empirical model and experimental data, although the method does depend on gathering lots of experimental data and it is not clear how transferable these correction factors would be to different sizes of rectangular splitter silencer, as well as different cross-sectional designs.

Clearly, it is preferable to develop a more comprehensive theoretical approach that is capable of capturing scattering at either end of the silencer, as well as computing the amplitudes of the modes propagating within the system. This has only recently been accomplished for bulk reacting splitter silencers in the absence of mean flow. For example, Kirby and Lawrie [15] used both analytic and numerical methods to analyse a splitter silencer of finite length, and Lawrie and Kirby [16] later presented an analytic technique that avoids root finding in the silencer section. The effect of fairings at either end of the silencer were later added by Kirby [17] using a point collocation technique, although it was assumed that these fairings were flat, rather than of a rounded "bull-nose" shape that is common in commercial silencer designs; mean flow effects were also omitted. The method of Kirby [17] does, however, represent the most complete model for splitter silencers currently available and so this article aims to retain the fairings at either end of the silencer but also to include chamfers on the fairings that are used to smooth out the mean flow.

The addition of mean flow into the model of a splitter silencer presents the problem of how to accommodate a change from low velocity in the inlet/outlet ducts to the higher velocities found in the airway between the parallel baffles. Clearly, it is desirable to avoid a sudden jump in fluid 
velocity over the inlet/outlet planes of the silencer so that one may properly enforce continuity conditions. In order to do this it is necessary to accommodate the non-uniform shape of the bullnose fairing at either end of the silencer. This is accomplished here for the acoustic part of the analysis by using the so-called hybrid method described by Kirby [3, 18], and this is reported in section 2. The experimental methodology is described in section 3, and in section 4 theoretical predictions are compared to experimental measurements both with and without mean flow. Following a discussion on the agreement between prediction and measurement a computationally efficient alternative approach to the hybrid method is then investigated in section 5 .

\section{THEORY}

The silencer geometry is separated into uniform and non-uniform regions, with modal expansions used for the uniform regions. A plan view of a splitter silencer is shown in Fig. 1. The silencer consists of three parallel baffles, with two baffles placed on opposite walls and one placed centrally, with a line of symmetry at $y=0$, see Fig. 1 . The analysis is restricted to three baffles because this configuration is very common commercially; however, the analysis that follows may readily be extended to any number of parallel baffles [17]. The mean gas flow is assumed to be steady, incompressible, inviscid and irrotational so that for the non-uniform regions a potential flow problem may be solved directly. These assumptions were also adopted by Eversman [19] in the study of sound radiation from a turbofan inlet, and these assumptions are justified on the basis that the flow velocities studied here are relatively low and that these approximations for the mean flow field will have a negligible effect on the acoustics of the problem. In Fig. 1 it is also assumed that the incident sound pressure field in $\mathrm{R}_{1}$ consists solely of a plane acoustic wave, which permits a two dimensional analysis only (x, y plane) [16]. 
The silencer is partitioned into five regions, bounded by planes A-D. Region $\mathrm{R}_{1}$ is assumed to have a uniform cross-section with a uniform mean flow of Mach number $\mathrm{M}_{1}$. Between planes $A$ and $B$ lies region $R_{A B}\left(=R_{2}+R_{3}+R_{4}\right)$ and here a potential mean flow field is present. In addition, region $\mathrm{R}_{3}$ is separated by a perforated plate from region $\mathrm{R}_{2}$, and region $\mathrm{R}_{3}$ contains a bulk reacting porous material in which the mean flow velocity is assumed to be negligible. The outer surface of region $\mathrm{R}_{4}$ that faces into $\mathrm{R}_{2}$ is the bull-nose fairing, which is assumed to be rigid and impervious to sound; the rest of region $\mathrm{R}_{4}$ is also assumed to be impervious to sound so that region $\mathrm{R}_{3}$ abuts onto a rigid wall. Planes $\mathrm{A}$ and $\mathrm{B}$ are drawn at locations sufficiently far from the bull-nose fairing to allow for the assumption of a uniform mean flow profile over each plane, so that in region $\mathrm{R}_{5}$ a uniform mean flow of Mach number $\mathrm{M}_{5}$ is present. The region lying between planes $\mathrm{C}$ and $\mathrm{D}\left(\mathrm{R}_{\mathrm{CD}}\right)$ is considered to be identical to region $\mathrm{R}_{\mathrm{AB}}$, with an equivalent incompressible potential mean flow field. Finally, $\mathrm{R}_{10}$ is assumed to be identical to $\mathrm{R}_{1}$, with a mean flow Mach number of $\mathrm{M}_{1}$, and an anechoic termination is assumed to be present at $x_{D}=\infty$.

In Fig. 2 a magnified plan view of the bull-nose fairing is shown, which consists of a central splitter of width $2 a$ and two parallel baffles of width $a$ placed against opposite walls; the gap between each splitter is $2 b$. The bull-nose fairings consist of a quarter circle of radius $r_{\mathrm{b}}$ and a flat section of width $w$ b. In order to compute the mean flow field around each fairing it is first assumed that this may be decoupled from the acoustic sound pressure field and that once the flow field has been calculated this may then be substituted into the acoustic analysis that follows. This approach has been used in the study of turbofan engines and the method of Eversman [19] is applied here. Accordingly, for the mean flow field the weighted residual formulation for a potential mean flow field in region $\mathrm{R}_{2}$ is written as [19] 


$$
\int_{R_{2}} \nabla \mathrm{W}^{\mathrm{T}} \cdot \nabla \varphi d R_{2}=\int_{\Gamma_{2}} \mathrm{~W}^{\mathrm{T}} \nabla \varphi \cdot \mathbf{n}_{2} d \Gamma_{2} .
$$

Here, $\mathrm{W}(x, y)$ is an element shape function and $\varphi$ is the velocity potential of the ambient fluid in region $\mathrm{R}_{2}$, so that the mean flow velocity $\mathbf{v}=\nabla \varphi$. The outer surface of $\mathrm{R}_{2}$ is denoted $\Gamma_{2}$ and $\mathbf{n}_{2}$ is the unit normal pointing out of $R_{2}$. Equation (1) is solved to find the mean flow profile in region $\mathrm{R}_{2}$ using the same finite element mesh as that used for the acoustic analysis that follows.

For the acoustic problem the presence of a non-uniform mean flow field means that one should use a more general velocity potential formulation, although the flow is also assumed to be steady and irrotational in order to simply the acoustic analysis that follows [20]. Accordingly, for region $q(q=1-10)$ the acoustic wave equation for steady irrotational flow, and for an isentropic equation of state, yields

$$
\nabla \cdot\left(\rho_{q} \nabla \phi_{q}\right)-\rho_{q}\left[\mathrm{i} \omega+\mathbf{v}_{q} \cdot \nabla\right] \frac{1}{c_{q}^{2}}\left[\mathrm{i} \omega+\mathbf{v}_{q} \cdot \nabla\right] \phi_{q}=0
$$

where $\rho_{q}$ is the ambient fluid density, $c_{q}$ is the speed of sound, $\phi_{q}$ is the acoustic velocity potential, and a time dependence of $e^{\mathrm{i} \omega t}$ is assumed throughout, with $t$ denoting time, $\omega$ radian frequency and $i=\sqrt{-1}$. Here, the acoustic particle velocity is defined as $u_{q}=\nabla \phi_{q}$, so that $p_{q}=-\rho_{q}\left(\partial / \partial t+\mathbf{v}_{q} \cdot \nabla\right) \phi_{q}[20]$. The assumption of incompressible flow permits Eq. (2) to be simplified to give 


$$
\nabla \cdot \nabla \phi_{q}-\left[\mathrm{i} k_{q}+\mathbf{M}_{q} \cdot \nabla\right]^{2} \phi_{q}=0
$$

where $k_{q}=\omega / c_{q}$ and $\mathbf{M}_{q}=\mathbf{v}_{q} / c_{q}$. Equation (3) is solved in section 2.1 using an eigenvalue analysis for the uniform regions of the silencer, which are then joined to a full finite element discretisation in the non-uniform regions in section 2.2.

\subsection{Finite element eigenvalue analysis}

The velocity potential for the uniform sections $\mathrm{R}_{1}, \mathrm{R}_{\mathrm{BC}}$ and $\mathrm{R}_{10}$ is expanded here as an infinite sum over the duct eigenmodes to give

$$
\begin{aligned}
& \phi_{1}(x, y)=\sum_{n=1}^{\infty} \mathrm{F}^{n} \Phi^{n}(y) \mathrm{e}^{-\mathrm{i} k_{0} \gamma_{i}^{n} x_{\mathrm{A}}}+\sum_{n=1}^{\infty} \mathrm{A}^{n} \Phi^{n}(y) \mathrm{e}^{-\mathrm{i} k_{0} \gamma_{r}^{n} x_{\mathrm{A}}} \\
& \phi_{\mathrm{BC}}(x, y)=\sum_{n=1}^{\infty} \mathrm{B}^{n} \Psi_{i}^{n}(y) \mathrm{e}^{-\mathrm{i} k_{0} \lambda_{i}^{n} x_{\mathrm{B}}}+\sum_{n=1}^{\infty} \mathrm{C}^{n} \Psi_{r}^{n}(y) \mathrm{e}^{-\mathrm{i} k_{0} \lambda_{r}^{n} x_{\mathrm{B}}} \\
& \phi_{10}(x, y)=\sum_{n=1}^{\infty} \mathrm{D}^{n} \Phi^{n}(y) \mathrm{e}^{-\mathrm{i} k_{0} \gamma_{i}^{n} x_{\mathrm{D}}} .
\end{aligned}
$$

Here, F, A, B, C and D are the modal amplitudes, $\gamma_{i}$ are the incident, and $\gamma_{r}$ the reflected (dimensionless) axial wavenumbers, and $\Phi_{i}(y)$ are the incident and $\Phi_{r}(y)$ the reflected eigenfunctions, in regions $\mathrm{R}_{1}$ and $\mathrm{R}_{10}$, respectively, where regions $\mathrm{R}_{1}$ and $\mathrm{R}_{10}$ are assumed to be identical with reflected waves omitted from region $\mathrm{R}_{10}$ in order to enforce an anechoic termination. For region $\mathrm{R}_{\mathrm{BC}}, \lambda_{i}$ are the incident and $\lambda_{r}$ the reflected (dimensionless) axial wavenumbers, and $\Psi_{i}(y)$ are the incident and $\Psi_{r}(y)$ the reflected eigenfunctions, respectively. 
The eigenvalue analysis for regions $\mathrm{R}_{1}$ and $\mathrm{R}_{10}$ is straightforward provided one assumes the outer surface of the duct is rigid and impervious to sound. This has been reported elsewhere in the literature, see for example Kirby [5], and so is not described further here. For the splitter section a general analysis for bulk reacting dissipative silencers that includes a uniform mean flow field has also appeared in the literature, see for example Astley and Cummings [14], and Kirby [3], therefore only a brief summary is presented here. To arrive at a governing eigenequation for region $\mathrm{R}_{\mathrm{BC}}$, it is necessary first to enforce continuity of pressure and normal particle displacement over the perforate $[3,4]$, and zero normal particle velocity over each hard wall boundary. If the (dimensionless) acoustic impedance of the perforate screen separating regions $\mathrm{R}_{5}$ and $\mathrm{R}_{6}$ is denoted $\zeta$, a finite element formulation delivers the following eigenequation

$$
\left[\begin{array}{cc}
\mathbf{0} & \mathbf{I} \\
-\mathbf{R}_{3}^{-1} \mathbf{R}_{1} & -\mathbf{R}_{3}^{-1} \mathbf{R}_{2}
\end{array}\right]\left\{\begin{array}{c}
\boldsymbol{\Psi} \\
\lambda \boldsymbol{\Psi}
\end{array}\right\}=\lambda\left\{\begin{array}{c}
\boldsymbol{\Psi} \\
\lambda \boldsymbol{\Psi}
\end{array}\right\}
$$

where, $\mathbf{I}$ is an identity matrix and the matrices $\mathbf{R}_{1}, \mathbf{R}_{2}$ and $\mathbf{R}_{3}$ are given by

$$
\begin{aligned}
& \mathbf{R}_{1} \boldsymbol{\Psi}=\left[\mathbf{H}_{5}-k_{0}^{2} \mathbf{L}_{5}\right] \boldsymbol{\Psi}_{5}+\beta\left[\mathbf{H}_{6}+\Gamma^{2} \mathbf{L}_{6}\right] \boldsymbol{\Psi}_{6}+\left(\mathrm{i} k_{0} M_{5} / \zeta\right)\left[\mathbf{L}_{\mathrm{p} 6}-\mathbf{L}_{\mathrm{p} 5}\right]\left\{\boldsymbol{\Psi}_{\mathrm{p} 6}-\boldsymbol{\Psi}_{\mathrm{p} 5}\right\} \\
& \mathbf{R}_{2} \boldsymbol{\Psi}=2 k_{0}^{2} M_{5} \mathbf{H}_{5} \boldsymbol{\Psi}_{5}+\left(2 \mathrm{i} k_{0} M_{5} / \zeta\right) \mathbf{L}_{\mathrm{p} 5}\left\{\boldsymbol{\Psi}_{\mathrm{p} 6}-\boldsymbol{\Psi}_{\mathrm{p} 5}\right\} \\
& \mathbf{R}_{3} \boldsymbol{\Psi}=k_{0}^{2}\left[1-M_{5}^{2}\right] \mathbf{H}_{5} \boldsymbol{\Psi}_{5}+\beta k_{0}^{2} \mathbf{H}_{6} \boldsymbol{\Psi}_{6}-\left(\mathrm{i} k_{0} M_{5}^{2} / \zeta\right) \mathbf{L}_{\mathrm{p} 5}\left\{\boldsymbol{\Psi}_{\mathrm{p} 6}-\boldsymbol{\Psi}_{\mathrm{p} 5}\right\} .
\end{aligned}
$$

In addition

$$
\begin{gathered}
\mathbf{H}_{5}=\int_{\mathrm{R}_{5}} \frac{\partial \mathrm{N}^{\mathrm{T}}}{\partial y} \cdot \frac{\partial \mathrm{N}}{\partial y} d \mathrm{R} \text { and } \mathbf{H}_{6}=\int_{\mathrm{R}_{6}} \frac{\partial \mathrm{N}^{\mathrm{T}}}{\partial y} \cdot \frac{\partial \mathrm{N}}{\partial y} d \mathrm{R} \\
\mathbf{L}_{5}=\int_{\mathrm{R}_{5}} \mathrm{~N}^{\mathrm{T}} \mathrm{N} d \mathrm{R} \text { and } \mathbf{L}_{6}=\int_{\mathrm{R}_{6}} \mathrm{~N}^{\mathrm{T}} \mathrm{N} d \mathrm{R}
\end{gathered}
$$




$$
\mathbf{L}_{\mathrm{p} 5}=\int_{\mathrm{S}_{\mathrm{p} 5}} \mathrm{~N}^{\mathrm{T}} \mathrm{N} d \mathrm{~S} \text { and } \mathbf{L}_{\mathrm{p} 6}=\int_{\mathrm{S}_{\mathrm{p} 6}} \mathrm{~N}^{\mathrm{T}} \mathrm{N} d \mathrm{~S}
$$

Here, $\mathrm{N}(y)$ is an element shape function so that the column vector $\boldsymbol{\Psi}$ holds the nodal values of the eigenfunction $\Psi(y)$. Vectors $\Psi_{\mathrm{p} 5}$ and $\boldsymbol{\Psi}_{\mathrm{p} 6}$ hold values of $\Psi(y)$ on the perforated screen, where $S_{p 5}$ and $S_{p 6}$ denote the outer surface of the perforated screen lying in regions $\mathrm{R}_{5}$ and $\mathrm{R}_{6}$, respectively (which for this problem are points located on opposite sides of the perforated screen). For region $\mathrm{R}_{3}$, the propagation constant of the porous material is denoted $\Gamma$ and the equivalent complex density $\rho_{m}$, where $\beta=\rho_{0} / \rho_{m}$, see Kirby [5]. Note that the ambient fluid properties of air in region $\mathrm{R}_{5}$ are denoted $\rho_{0}$ and $c_{0}$, so that $k_{0}=k_{5}$. Equation (7) is solved for $n_{\mathrm{BC}}$ incident and $n_{\mathrm{BC}}$ reflected eigenmodes and their associated eigenvectors. Following this the imaginary part of the eigenmodes are sorted into ascending order. Note that in this eigenvalue analysis the perforated screen is backed by a porous material; this material is assumed to damp down any hydrodynamic modes that may be present in the silencer so that these modes may be omitted from the analysis that follows without loss of accuracy [5].

\subsection{Hybrid finite element method for non-uniform regions}

In order to condense the analysis that follows, regions $\mathrm{R}_{\mathrm{AB}}$ and $\mathrm{R}_{\mathrm{CD}}$ are assumed to be identical mirror images of one another and so the analysis is restricted to RAB. Furthermore, a finite element analysis for a dissipative silencer with mean flow has been reported previously $[3,6]$ and so only a brief recap is reported here, although previous models are revised in this section to accommodate the non uniform mean flow field. The airway in region $\mathrm{R}_{\mathrm{AB}}$ is denoted $R_{2}$ and the acoustic velocity potential in region $R_{2}$ is approximated using the shape function $\mathrm{W}(x, y)$ so that formulation for region $\mathrm{R}_{2}$ yields, 


$$
\begin{aligned}
\int_{\mathrm{R}_{2}}\left\{\nabla \mathrm{W}^{\mathrm{T}} \cdot \mathbf{T}_{2}\right. & \left.\nabla \phi_{2}+2 \mathrm{i} k_{0} \mathrm{~W}^{\mathrm{T}}\left[\mathbf{M}_{2} \cdot \nabla \phi_{2}\right]-k_{0}^{2} \mathrm{~W}^{\mathrm{T}} \phi_{2}\right\} d \mathrm{R}_{2} \\
& =\int_{\Gamma_{2}} \mathrm{~W}^{\mathrm{T}}\left[1-\mathrm{M}_{2 x}^{2}\right] \frac{\partial \phi_{2}}{\partial x} \cdot \mathbf{n}_{2 x} d \Gamma_{2}+\int_{\Gamma_{2}} \mathrm{~W}^{\mathrm{T}}\left[1-\mathrm{M}_{2 y}^{2}\right] \frac{\partial \phi_{2}}{\partial y} \cdot \mathbf{n}_{2 y} d \Gamma_{2},
\end{aligned}
$$

where, $\mathbf{M}_{2}=\left[\begin{array}{ll}\mathrm{M}_{2 x} & \mathrm{M}_{2 y}\end{array}\right]^{\mathrm{T}}$, and

$$
\mathbf{T}_{2}=\left[\begin{array}{cc}
1-\mathrm{M}_{2 x}^{2} & -\mathrm{M}_{2 x} \mathrm{M}_{2 y} \\
-\mathrm{M}_{2 x} \mathrm{M}_{2 y} & 1-\mathrm{M}_{2 y}^{2}
\end{array}\right]
$$

In addition, $\mathbf{n}_{2 x}$ and $\mathbf{n}_{2 y}$ are the unit normals pointing out of surface $\Gamma_{2}$ in the $x$ and $y$ directions, respectively. Note that Eq. (14) is similar to that reported by Eversman [19], although the assumption of incompressible flow allows for the removal of an extra term from the integral over $\Gamma_{2}$. Similarly, for region $\mathrm{R}_{3}$

$$
\int_{\mathrm{R}_{3}}\left\{\nabla \mathrm{W}^{\mathrm{T}} \cdot \nabla \phi_{3}+\Gamma^{2} \mathrm{~W}^{\mathrm{T}} \phi_{3}\right\} d \mathrm{R}_{3}=\int_{\Gamma_{3}} \mathrm{~W}^{\mathrm{T}} \frac{\partial \phi_{3}}{\partial x} \cdot \mathbf{n}_{3 x} d \Gamma_{3}+\int_{\Gamma_{3}} \mathrm{~W}^{\mathrm{T}} \frac{\partial \phi_{3}}{\partial y} \cdot \mathbf{n}_{3 y} d \Gamma_{3} .
$$

Equations (14) and (16) may be joined together by enforcing continuity of pressure and displacement over the perforated screen $[3,4]$, to give

$$
\begin{aligned}
\int_{\mathrm{R}_{2}}\left\{\nabla \mathrm{W}^{\mathrm{T}} \cdot \mathbf{T}_{2}\right. & \left.\nabla \phi_{2}+2 \mathrm{i} k_{0} \mathrm{~W}^{\mathrm{T}}\left[\mathbf{M}_{2} \cdot \nabla \phi_{2}\right]-k_{0}^{2} \mathrm{~W}^{\mathrm{T}} \phi_{2}\right\} d \mathrm{R}_{2} \\
& +\beta \int_{\mathrm{R}_{3}}\left\{\nabla \mathrm{W}^{\mathrm{T}} \cdot \nabla \hat{\phi}_{3}+\Gamma^{2} \mathrm{~W}^{\mathrm{T}} \hat{\phi}_{3}\right\} d \mathrm{R}_{3} \\
& =\int_{\Gamma_{\mathrm{A}}} \mathrm{W}^{\mathrm{T}}\left[1-\mathrm{M}_{1}^{2}\right] \frac{\partial \phi_{2}}{\partial x} \cdot \mathbf{n}_{\mathrm{A}} d \Gamma_{\mathrm{A}}+\int_{\Gamma_{\mathrm{B}}} \mathrm{W}^{\mathrm{T}}\left[1-\mathrm{M}_{5}^{2}\right] \frac{\partial \phi_{2}}{\partial x} \cdot \mathbf{n}_{\mathrm{B}} d \Gamma_{\mathrm{B}} \\
& +\beta \int_{\Gamma_{\mathrm{B}}} \mathrm{W}^{\mathrm{T}} \frac{\partial \hat{\phi}_{3}}{\partial x} \cdot \mathbf{n}_{\mathrm{B}} d \Gamma_{\mathrm{B}} \\
& +\frac{\mathrm{i} k_{0}}{\zeta} \int_{\Gamma_{\mathrm{p} 2}} \mathrm{~W}^{\mathrm{T}}\left[\hat{\phi}_{3}-\phi_{2}+2 \mathrm{i} \frac{\mathrm{M}_{5}}{k_{0}} \frac{\partial \phi_{2}}{\partial x}+\frac{\mathrm{M}_{5}^{2}}{k_{0}} \frac{\partial^{2} \phi_{2}}{\partial^{2} x}\right] d \Gamma_{\mathrm{p} 2} \\
& -\frac{\mathrm{i} k_{0}}{\zeta} \int_{\Gamma_{\mathrm{p} 3}} \mathrm{~W}^{\mathrm{T}}\left[\hat{\phi}_{3}-\phi_{2}\right] d \Gamma_{\mathrm{p} 3}
\end{aligned}
$$


Here, $\Gamma_{A}$ and $\Gamma_{B}$ denote the surface of planes $A$ and $B$, respectively, and $\Gamma_{\mathrm{p} 2}$ and $\Gamma_{\mathrm{p} 3}$ denote the surface of the perforates that lie in region $\mathrm{R}_{2}$, or region $\mathrm{R}_{3}$, respectively. The unit normals in the $x$ direction over planes $\mathrm{A}$ and $\mathrm{B}$ are denoted by $\mathbf{n}_{\mathrm{A}}$ and $\mathbf{n}_{\mathrm{B}}$, respectively, and $\phi_{3}=$ $\beta \hat{\phi}_{3}$. The hybrid numerical method enforces continuity of displacement in the surface integrals in Eq. (17) and continuity of pressure is enforced separately. This delivers the following system of equations:

$$
\begin{gathered}
\mathbf{M}_{1 r} \mathbf{A}-\mathbf{Q}_{r} \boldsymbol{\phi}_{2 \mathrm{~A}}=-\mathbf{M}_{1 r} \mathbf{F} \\
\mathbf{K}_{2} \boldsymbol{\phi}_{2}+\mathbf{K}_{3} \widehat{\boldsymbol{\phi}}_{3}-\left[\mathbf{V}_{2}-\mathbf{V}_{3}\right]\left\{\widehat{\boldsymbol{\phi}}_{\mathrm{p} 3}-\boldsymbol{\phi}_{\mathrm{p} 2}\right\}-\mathbf{Q}_{r}^{\mathrm{T}} \mathbf{A}+\mathbf{R}_{i}^{\mathrm{T}} \mathbf{B}+\mathbf{R}_{r}^{\mathrm{T}} \mathbf{C}=\mathbf{Q}_{i}^{\mathrm{T}} \mathbf{F} . \\
-\mathbf{M}_{2 i} \mathbf{B}-\mathbf{M}_{2 r} \mathbf{C}+\mathbf{R}_{i} \boldsymbol{\phi}_{2 \mathrm{~B}}=\mathbf{0} .
\end{gathered}
$$

Similarly, for $\mathrm{R}_{\mathrm{CD}}\left(=\mathrm{R}_{7}+\mathrm{R}_{8}\right)$,

$$
\begin{gathered}
\mathbf{M}_{7 i} \mathbf{E}_{i} \mathbf{B}+\mathbf{M}_{7 r} \mathbf{E}_{r} \mathbf{C}-\mathbf{X}_{r} \boldsymbol{\phi}_{7 \mathrm{C}}=\mathbf{0} \\
\mathbf{K}_{7} \boldsymbol{\phi}_{7}+\mathbf{K}_{8} \widehat{\boldsymbol{\phi}}_{8}-\left[\mathbf{V}_{7}-\mathbf{V}_{8}\right]\left\{\widehat{\boldsymbol{\phi}}_{\mathrm{p} 8}-\boldsymbol{\phi}_{\mathrm{p} 7}\right\}-\mathbf{X}_{i}^{\mathrm{T}} \mathbf{E}_{i} \mathbf{B}-\mathbf{X}_{r}^{\mathrm{T}} \mathbf{E}_{r} \mathbf{C}+\mathbf{Y}_{i}^{\mathrm{T}} \mathbf{D}=\mathbf{0} \\
-\mathbf{M}_{10 i} \mathbf{D}+\mathbf{Y}_{i} \boldsymbol{\phi}_{7 \mathrm{D}}=\mathbf{0} .
\end{gathered}
$$

Here, the velocity potential is also discretised using the shape function $\mathrm{W}(x, y)$ so that the column vector $\boldsymbol{\phi}_{q}$ holds the values of the velocity potential $\phi_{q}(x, y)$ at individual nodes in region $q$. Vector $\boldsymbol{\phi}_{\mathrm{p} q}$ holds values for nodes at a perforate in region $q$, and $\boldsymbol{\phi}_{2 \mathrm{~A}, \mathrm{~B}}$ and $\boldsymbol{\phi}_{7 \mathrm{C}, \mathrm{D}}$ hold values on the inlet and outlet planes of each non uniform region. The other matrices that make up this system of equations are given in the Appendix. The problem is solved by 
joining together Eqs. (18) - (23) and solving them simultaneously. In order to do this it is convenient to write

$$
\mathbf{G} \boldsymbol{\phi}_{\mathrm{AB}}=\mathbf{K}_{2} \boldsymbol{\phi}_{2}+\mathbf{K}_{3} \boldsymbol{\phi}_{3}-\left[\mathbf{V}_{2}-\mathbf{V}_{3}\right]\left\{\widehat{\boldsymbol{\phi}}_{\mathrm{p} 3}-\boldsymbol{\phi}_{\mathrm{p} 2}\right\}
$$

and to subdivide this matrix into the component velocity potentials for each region, so that

$$
\mathbf{G}_{\mathrm{AB}}=\left[\begin{array}{ccc}
\mathbf{G}_{\mathrm{AA}} & \mathbf{G}_{\mathrm{Ae}} & \mathbf{G}_{\mathrm{AB}} \\
\mathbf{G}_{\mathrm{eA}} & \mathbf{G}_{\mathrm{ee}} & \mathbf{G}_{\mathrm{eB}} \\
\mathbf{G}_{\mathrm{BA}} & \mathbf{G}_{\mathrm{Be}} & \mathbf{G}_{\mathrm{BB}}
\end{array}\right]\left\{\begin{array}{c}
\boldsymbol{\phi}_{\mathrm{A}} \\
\boldsymbol{\phi}_{\mathrm{ABe}} \\
\boldsymbol{\phi}_{\mathrm{B}}
\end{array}\right\} .
$$

Similarly,

$$
\widetilde{\mathbf{G}} \boldsymbol{\phi}_{\mathrm{CD}}=\mathbf{K}_{7} \boldsymbol{\phi}_{7}+\mathbf{K}_{8} \boldsymbol{\phi}_{8}-\left[\mathbf{V}_{7}-\mathbf{V}_{8}\right]\left\{\widehat{\boldsymbol{\phi}}_{\mathrm{p} 8}-\boldsymbol{\phi}_{\mathrm{p} 7}\right\}
$$

which yields

$$
\widetilde{\mathbf{G}} \boldsymbol{\phi}_{\mathrm{CD}}=\left[\begin{array}{ccc}
\mathbf{G}_{\mathrm{CC}} & \mathbf{G}_{\mathrm{Ce}} & \mathbf{G}_{\mathrm{CD}} \\
\mathbf{G}_{\mathrm{eC}} & \mathbf{G}_{\mathrm{ee}} & \mathbf{G}_{\mathrm{eD}} \\
\mathbf{G}_{\mathrm{DC}} & \mathbf{G}_{\mathrm{De}} & \mathbf{G}_{\mathrm{DD}}
\end{array}\right]\left\{\begin{array}{c}
\boldsymbol{\phi}_{\mathrm{C}} \\
\boldsymbol{\phi}_{\mathrm{CDe}} \\
\boldsymbol{\phi}_{\mathrm{D}}
\end{array}\right\} .
$$

Matrix $\mathbf{G}_{m n}$ has order $n_{m} \times n_{n}$, where $n_{\mathrm{A}}$ and $n_{\mathrm{B}}$ are the number of nodes on $\Gamma_{\mathrm{A}}$ and $\Gamma_{\mathrm{B}}$, respectively (with $m_{\mathrm{A}} \leq n_{\mathrm{A}}$, and $m_{\mathrm{B}} \leq n_{\mathrm{B}}$ ); $n_{\mathrm{AB}}$ is the number of nodes in region $\mathrm{R}_{\mathrm{AB}}$, and $n_{e}$ is the number of nodes in region $\mathrm{R}_{\mathrm{AB}}$ that do not lie on $\Gamma_{\mathrm{A}}$ and $\Gamma_{\mathrm{B}}$, so that $n_{e}=n_{\mathrm{AB}}-$ $n_{A}-n_{B}$. The value for the velocity potential at those nodes in region $\mathrm{R}_{\mathrm{AB}}$ that do not lie on $\Gamma_{\mathrm{A}}$ and $\Gamma_{\mathrm{B}}$ are held in matrix $\boldsymbol{\phi}_{\mathrm{ABe}}$. Similar arguments also apply to $\widetilde{\mathbf{G}}_{m n}$. Equations (24) (27) are now joined together to give 


$$
\left[\begin{array}{cccccccccc}
\mathbf{M}_{1 r} & -\mathbf{Q}_{r} & \mathbf{0} & \mathbf{0} & \mathbf{0} & \mathbf{0} & \mathbf{0} & \mathbf{0} & \mathbf{0} & \mathbf{0} \\
-\mathbf{Q}_{r}^{\mathrm{T}} & \mathbf{G}_{\mathrm{AA}} & \mathbf{G}_{\mathrm{Ae}} & \mathbf{G}_{\mathrm{AB}} & \mathbf{0} & \mathbf{0} & \mathbf{0} & \mathbf{0} & \mathbf{0} & \mathbf{0} \\
\mathbf{0} & \mathbf{G}_{\mathrm{eA}} & \mathbf{G}_{\mathrm{ee}} & \mathbf{G}_{\mathrm{eB}} & \mathbf{0} & \mathbf{0} & \mathbf{0} & \mathbf{0} & \mathbf{0} & \mathbf{0} \\
\mathbf{0} & \mathbf{G}_{\mathrm{BA}} & \mathbf{G}_{\mathrm{Be}} & \mathbf{G}_{\mathrm{BB}} & \mathbf{R}_{i}^{\mathrm{T}} & \mathbf{R}_{r}^{\mathrm{T}} \mathbf{E}_{r} & \mathbf{0} & \mathbf{0} & \mathbf{0} & \mathbf{0} \\
\mathbf{0} & \mathbf{0} & \mathbf{0} & \mathbf{R}_{i} & -\mathbf{M}_{2 i} & -\mathbf{M}_{2 r} \mathbf{E}_{r} & \mathbf{0} & \mathbf{0} & \mathbf{0} & \mathbf{0} \\
\mathbf{0} & \mathbf{0} & \mathbf{0} & \mathbf{0} & -\mathbf{M}_{7 i} \mathbf{E}_{i} & \mathbf{M}_{7 r} & -\mathbf{X}_{r} & \mathbf{0} & \mathbf{0} & \mathbf{0} \\
\mathbf{0} & \mathbf{0} & \mathbf{0} & \mathbf{0} & -\mathbf{X}_{i}^{\mathrm{T}} \mathbf{E}_{\mathbf{i}} & -\mathbf{X}_{r}^{\mathrm{T}} & \mathbf{G}_{\mathrm{CC}} & \mathbf{G}_{\mathrm{Ce}} & \mathbf{G}_{\mathrm{CD}} & \mathbf{0} \\
\mathbf{0} & \mathbf{0} & \mathbf{0} & \mathbf{0} & \mathbf{0} & \mathbf{0} & \mathbf{G}_{\mathrm{eC}} & \mathbf{G}_{\mathrm{ee}} & \mathbf{G}_{\mathrm{eD}} & \mathbf{0} \\
\mathbf{0} & \mathbf{0} & \mathbf{0} & \mathbf{0} & \mathbf{0} & \mathbf{0} & \mathbf{G}_{\mathrm{DC}} & \mathbf{G}_{\mathrm{De}} & \mathbf{G}_{\mathrm{DD}} & \mathbf{Y}_{i}^{\mathrm{T}} \\
\mathbf{0} & \mathbf{0} & \mathbf{0} & \mathbf{0} & \mathbf{0} & \mathbf{0} & \mathbf{0} & \mathbf{0} & \mathbf{Y}_{i} & -\mathbf{M}_{10 i}
\end{array}\right]\left\{\begin{array}{c}
\mathbf{A} \\
\boldsymbol{\phi}_{\mathrm{A}} \\
\boldsymbol{\phi}_{\mathrm{ABe}} \\
\boldsymbol{\Phi}_{\mathrm{B}} \\
\mathbf{B} \\
\tilde{\mathbf{C}} \\
\boldsymbol{\phi}_{\mathrm{C}} \\
\boldsymbol{\Phi}_{\mathrm{CDe}} \\
\boldsymbol{\phi}_{\mathrm{D}} \\
\mathbf{D}
\end{array}\right\}=\left\{\begin{array}{c}
-\mathbf{M}_{1 r} \mathbf{F} \\
\mathbf{Q}_{i}^{\mathrm{T}} \mathbf{F} \\
\mathbf{0} \\
\mathbf{0} \\
\mathbf{0} \\
\mathbf{0} \\
\mathbf{0} \\
\mathbf{0} \\
\mathbf{0} \\
\mathbf{0}
\end{array}\right\}
$$

where $\mathbf{C}=\tilde{\mathbf{C}} \mathbf{E}_{r}$. Equation (28) is solved to obtain the unknown modal amplitudes and the velocity potentials in regions $R_{A B}$ and $R_{C D}$, once the appropriate modal amplitudes of the incident sound field have been specified. Using the model outlined above it is possible to assign a range of different characteristics to the sound source and to include sources which drive higher order modes, see for example those discussed by Mechel [21] and Kirby and Lawrie [15]; however, the predictions obtained here will be compared against experimental data taken under laboratory conditions that assume plane wave excitation. Accordingly, the inlet modal amplitude is specified as $\mathrm{F}^{0}=1 /\left(1+\mathrm{M}_{1}\right)$, and $\mathrm{F}^{n}=0$ for $n>0$; this choice for $\mathrm{F}^{0}$ sets the inlet sound power equal to unity.

Silencer performance is readily expressed in terms of transmission loss (or insertion loss, which will be discussed later), and is defined as the ratio of the transmitted to incident sound powers. Following the setting of the inlet sound power equal to unity, the transmission loss, in decibels, is given as [22]

$$
\mathrm{TL}=-10 \log _{10} \sum_{n=0}^{n_{I}}\left[\frac{\mathrm{M}_{1}+\left(1-\mathrm{M}_{1}^{2}\right) \gamma_{i}^{n}}{\left(1-\mathrm{M}_{1} \gamma_{i}^{n}\right)^{2}}\right] \frac{I_{i}^{n}\left|\mathrm{D}^{n}\right|^{2}}{I_{i}^{0}}
$$

where $I_{i}^{n}=\int_{\Gamma_{\mathrm{D}}}\left|\Phi_{i}^{n}(y)\right|^{2} d y$, and $n_{I}$ is the number of propagating modes in region $\mathrm{R}_{10}$. Note that even though the excitation is restricted to a plane wave only, Eq. (29) accommodates all 
higher order modes that propagate downstream of the silencer. However, Eq. (29) assumes that there is no transfer of energy between propagating acoustic modes and hydrodynamic modes that may form on shear layers at the exit from the silencer.

\section{EXPERIMENT}

A measurement methodology for splitter silencers is outlined in the European Standard EN ISO 7235 [23], and in the U.S. by ASTM E 477 [24]. Both standards seek to measure the insertion loss of a silencer with and without mean flow. An interesting difference between the two standards is that ISO 7235 specifies a "modal filter" to be placed between the sound source and the test silencer, whereas this is not included in E 477 . The modal filter in ISO 7235 is intended to damp down higher order modes emitted by the sound source and to deliver an incident sound field with a "dominating plane wave mode in the test duct in front of the test silencer" [23]. This approach has significant advantages when attempting to compare theory and experiment because it unambiguously specifies the modal characteristics of the incident sound field. Accordingly, ISO 7235 is used here to measure the IL of four splitter silencers.

The material used in the test silencers is rock wool and this is normally purchased in the form of large "slabs" of material, which are then dropped into a steel carcass that forms each baffle. The rock wool is separated from the silencer airway by a sheet of perforated steel, which runs the length of each baffle and abuts onto the bull nose fairings at either end, see Figs. 1 and 2. The rock wool is treated as an equivalent fluid that is homogeneous and isotropic, which permits the use of the standard Delany and Bazley approach for characterising the bulk acoustic properties of a bulk fibrous material. These properties were 
measured in an impedance tube using the two microphone technique [25] and for the normalised propagation constant, this gives [26]

$$
\widehat{\Gamma}=0.2722 \xi^{-0.4718}+\mathrm{i}\left[1+0.2432 \xi^{-0.4326}\right]
$$

and for the normalised complex density

$$
\hat{\rho}=-\widehat{\Gamma}\left\{0.1591 \xi^{-0.5328}+\mathrm{i}\left[1+0.1316 \xi^{-0.5398}\right]\right\}
$$

Here $\hat{\Gamma}=\Gamma / k_{0}, \hat{\rho}=1 / \beta$ and $\xi=\rho_{0} f / \Theta$, where $f$ is frequency and $\Theta$ is the flow resistivity of the porous material. The flow resistivity of the porous material was measured in accordance with ISO 29053 [27], which gives $\sigma=1881 \mathrm{~Pa} \mathrm{~s} / \mathrm{m}^{2}$. Note that the bulk acoustic properties and flow resistivity of rock wool were measured for random samples of the material in which any preferential alignment of the fibres was removed by breaking up the material before testing. Following previous articles by the first author, a semi-empirical correction is used here to avoid inconsistencies in the Delany and Bazley curve fitting formulae at low frequencies $[17,28]$. This yields a value of 2.48 for the steady flow tortuosity at a transition value of 0.01085 , see also [4].

For the perforated screen that lies between the airway and the rock wool, the semi-empirical model of Kirby and Cummings is used [29]. This model is further modified using the method suggested by Denia et al. [30], to give

$$
\zeta=\left[\zeta^{\prime}+\mathrm{i} 0.425 k d(\hat{\rho}-1) \mathrm{F}(\sigma)\right] / \sigma
$$

with

$$
\mathrm{F}(\sigma)=1-1.06 \sigma^{0.5}+0.17 \sigma^{1.5}
$$


Here, $\sigma$ is the open area porosity of the perforated screen, $d$ is the hole diameter and $\zeta^{\prime}$ is the orifice impedance measured in the absence of a porous material. Values for $\zeta^{\prime}$ in the presence of a mean grazing flow are widely available in the literature, although the data measured by Kirby and Cummings is used here [29]. For each silencer studied, $d=3 \mathrm{~mm}$, $\sigma=27 \%$ and the thickness of the perforated screen $t_{p}=1.6 \mathrm{~mm}$.

\section{RESULTS}

Comparisons between predicted and measured data are presented in this section, both with and without mean flow. It is sensible first to examine comparisons without flow in order to explore any differences between theory and measurement without the added complication of mean flow. Insertion loss measurements were carried out for over 60 different silencers and so only a small sample of the data measured is reported here, although this data has been carefully chosen to be representative of the wider range of results that have been obtained. The dimensions of the test silencers to be investigated are given in Table 1, where the percentage open area of the silencer $\Delta=b /(a+b)$. In the predictions that follow, the transverse finite element mesh for each uniform region uses three noded line elements, and for the non uniform regions eight noded quadrilateral elements are used. Before generating the final predictions for each silencer design the effect of changing the location of planes A-D was investigated by studying the mean flow field and the convergence of the acoustic problem. In general it was found that placing planes A and D a distance of $4(a+b)$ away from the front/end of the bull nose fairing, and placing planes B and C a distance of $2(a+$ b) $+r_{a}$ away provided a good compromise between the number of elements required and the need to deliver a uniform flow field over each plane. 
In the results that follow, silencer IL is presented, which is the difference between the sound power level measured with and without the test silencer in place. This is different to the silencer transmission loss (TL), which is defined as the difference between the sound powers incident and transmitted through the silencer. ISO 7235 notes that "for measurements according to this International Standard, the insertion loss of a silencer equals the transmission loss." Accordingly, in the discussions that follow we shall refer to the silencer IL rather than TL, largely because the silencer industry prefers IL.

\subsection{Silencer performance in the absence of mean flow}

In Fig. 4, the predicted and measured IL is compared for silencer A for one third octave bands. The predictions in Fig. 4 were generated using 9446 degrees of freedom, which are chosen to provide good convergence (to one decimal place) for the IL at the highest (centre) frequency of $8 \mathrm{kHz}$. Figure 4 generally shows good agreement between prediction and measurement over the entire frequency range. Discrepancies below $100 \mathrm{~Hz}$ are thought to be caused by experimental error, which is likely to be caused by problems with the anechoic terminations at very low frequencies. Whilst a slight over prediction is seen at peak IL, Fig. 4 demonstrates that it is possible to predict successfully the performance of a splitter silencer over a wide frequency range. This is also observed for silencers B and C in Figs. 5 and 6, respectively, although some discrepancy is again observed at peak IL values. The sharp peak in IL loss seen in each figures is caused by energy transferring from the silencer section into higher order modes that have suddenly cut-on in the outlet duct $\left(\mathrm{R}_{10}\right)$. The frequency at which this peak appears is therefore dictated by the overall width of the outlet duct and not, for example, by the length of the silencer, see Kirby and Lawrie for a more detailed discussion [15]. 
Silencers A and B have a percentage open area of $\Delta=50 \%$ and for silencer C $\Delta=38.5 \%$. It was generally found that for higher percentage open areas the agreement between prediction and measurement was comparable to that shown in Figs. 4-6. However, if one studies silencers with a lower percentage open area then problems with over prediction at higher frequencies may occur. For example, in Fig. 7 the IL for silencer D is shown. This silencer has a very low percentage open area of $\Delta=20 \%$ and represents the worst case scenario of all of the silencers measured (including those not shown here). Clearly, agreement between prediction and experiment is less successful at medium to high frequencies, although the low frequency behaviour is still captured reasonably well. This problem with predicting medium to high frequency performance for silencers with a low percentage open area has generally been found to appear once the percentage open area drops below about $30-35 \%$. This will be discussed further in section 5 .

\subsection{Silencer performance with mean flow}

During the course of the experimental programme it was found to be a significant challenge to undertake meaningful measurements when mean flow is present. During the commissioning of the test rig problems were apparent with background (flow generated) noise and this limited the face velocities for which experiments were possible. A particular problem was that the modal filter specified by ISO 7235 [23] impacted upon the signal to noise ratio in the medium frequency range, which coincides with high attenuations for the silencers being studied. Moreover, when conducting experiments it is desirable to see a significant influence on silencer performance when mean flow is present. This generally demands the study of larger silencers, but a combination of the modal filter and higher silencer attenuation serves to further limit the available signal to noise ratio. Accordingly, 
during the testing programme it was found to be impossible to combine large silencers with relatively large face velocities without generating problems with the signal to noise ratio. These problems may be addressed by, for example, lowering the attenuation of the modal filter and/or changing the sound source, but it was felt that this would potentially incur significant extra costs with no guarantee that a solution could readily be found. Accordingly, the data reported here is restricted to lower face velocities.

In Fig. 8 predictions are compared against experiment for silencer A with a face velocity of $3.7 \mathrm{~m} / \mathrm{s}$ and it is not surprising to see that the addition of mean flow has little measurable effect on silencer performance (the airway Mach number is $M_{5}=0.022$ ). In Fig. 9 predictions are compared against experiment for silencer D with a face velocity of $2.95 \mathrm{~m} / \mathrm{s}$. Silencer D has a lower percentage open area when compared to silencer A and so for this silencer the Mach number in the airway increases to $M_{5}=0.043$. Thus, in Figs. 8 and 9 a slight lowering of IL is seen at lower frequencies and this behaviour is similar to that observed by Cummings and Sormaz [10], and Brandstätt et al. [12]. However, in Figs. 8 and 9 , only a limited investigation into the influence of mean flow has been possible because of the limitation of the experimental methodology. Following a number of additional measurements covering a number of different silencer geometries it was concluded that the effect of mean flow on silencer IL was small to negligible for those face velocities for which it was possible to adhere to ISO 7235 [23]. This does not, however, prevent further investigations using the theoretical model and so the predicted influence of mean flow on the IL for silencers A-D is shown in Figs. 10-13, respectively. Here, the IL is plotted for different airway mean flow Mach numbers of $M_{5}= \pm 0.1$ and \pm 0.2 . It is evident in Figs. 10-13 that the mean flow has a relatively systematic effect on silencer performance at low frequencies, with silencer performance reducing when the mean flow is in the same direction 
as the sound propagation, and increasing when the mean flow is reversed. At higher frequencies the effect of mean flow is seen to reduce and no obvious trend in performance is observed. In fact from these figures one may conclude that it is possible to neglect the influence of mean flow on silencer performance at higher frequencies because one is unlikely to be able to measure the IL sufficiently accurately to resolve the differences seen in IL, even at high Mach numbers. This has ramifications for silencer modelling because neglecting mean flow reduces the computational time of the eigensolution for the silencer section and this saving is likely to be significant for those higher frequencies encountered here.

\section{DISCUSSION}

The nature of the discrepancy between prediction and experiment seen in fig. 7 has been observed before by Mechel [21] and also Kirby [17] who used Mechel's measured data to demonstrate good agreement for larger percentage open areas, but for an open area of $33 \%$ poor agreement was observed at higher frequencies. Clearly the same problem persists with the current data, although the agreement between prediction and experiment is much better in the current study when compared to that presented by Kirby [17]. It is, however, difficult to be certain as to why these problems persist, although it is likely that they are caused by a combination of theoretical and experimental inaccuracies. For the experimental measurements, Kirby [17] proposed that problems may be caused by structural flanking transmission in which the incident sound energy bypasses the silencer and breaks back into the duct on the far side of the silencer. However, the limiting insertion loss for the experimental facility was significantly above the values of IL measured here and so flanking transmission should not be a problem. Another possible source of error is in the assumption that the measured IL is the same as the predicted TL. This assumption requires that the sound 
source in the measurements provides an identical output, both with and without the silencer present. However, it is possible that standing waves may be set up between the silencer and the sound source and/or the modal filter. Here, one may expect significant levels of energy to be reflected back towards the sound source by a silencer with a low percentage open area. It is possible that this reflected energy may affect the output of the sound source, as well as alter the nature of the sound field incident on the test silencer. This problem was also noted by Roland [31] and it is possible that these reflections from silencers with lower percentage open areas are causing problems with the experimental measurements.

The other source of discrepancy lies with the theoretical predictions. In the model it is necessary to assume uniformity of the material packing as well as the material properties, which is unlikely to be the case in a real silencer. Moreover, the silencer predictions are sensitive to the impedance of the perforate and it is difficult to quantify accurately the impedance of the perforate over such a wide frequency range. The perforate impedance also depends strongly on the conditions very close to the perforate [29], and in the model it is assumed that a uniform density for the material is always adjacent to the perforate. Again, this is unlikely to be true in practice and this may affect the actual perforate impedance. Nevertheless, it is interesting to note that generally good agreement can be found between prediction and experiment at higher open areas. Further, one would expect that any systematic problems with the material specification, and/or perforate impedance, would show up across all of the silencer measurements, whereas this is not the case in practice. Therefore, it is difficult to identify problems with the theoretical model that would apply only to those silencers of low percentage open area and at high frequencies, assuming of course that the theoretical model is fully converged (which has been extensively verified). 
Modelling large dissipative silencer performance up to a frequency of $8 \mathrm{kHz}$ presents a difficult problem and one has to be realistic regarding the level of agreement that may be expected when comparing predictions with experimental measurements, especially as the experimental measurements are difficult to undertake. In view of this the theoretical model appears to perform well under most conditions and over a wide range of different geometries, most of which have not been shown here. Furthermore, it is common for silencer manufacturers to be more concerned about low frequency performance and here the theoretical model performs well. Therefore, it appears appropriate to conclude that, within the bounds of experimental and theoretical uncertainty, the theoretical model provides a reliable guide to the true performance of a splitter silencer, at least for plane wave excitation.

\subsection{Lowering computational demands}

The theoretical model developed in section 2 is designed to accommodate the geometry of a typical splitter silencer. This includes the bull nose fairings at either end of the silencer, as well as the chamfer that is normally present to smooth the air flow. However, modelling the bull-nose fairings is computationally expensive and so it is interesting to see if one can develop a simpler model that will lower computational demands whilst at the same time maintaining prediction accuracy. The most obvious simplification is to ignore the chamfers and to use a flat fairing at either end of the silencer. This approach was used by Kirby [17] in the absence of mean flow, and it has the advantage of allowing the two dimensional finite element mesh between planes $\mathrm{A}$ and $\mathrm{B}$, and $\mathrm{C}$ and $\mathrm{D}$ to be removed. This permits the use of point collocation to enforce continuity conditions over the inlet and outlet planes of the silencer, which will considerably lower computational expenditure when compared to the hybrid method described earlier. The disadvantage of this approach is that one enforces the acoustic continuity conditions over a jump in the mean flow profile. To see the effect of this 
approximation, the predicted IL for silencer A is compared with and without a chamfer, and this is done without mean flow in Fig 14, and with mean flow in Fig. 15. Here the predictions with a chamfer are obtained using the method described in section 2 , with values of $r_{b}=0.025$ and $r_{b}=0.05$; without the chamfer the point collocation method of Kirby [3, 17] is adopted, with continuity of pressure and displacement enforced over the ends of the silencer following the addition of mean flow, see Kirby [18]. In Fig. 14 it can be seen that when no mean flow is present the chamfer on the fairings has little effect on the IL of silencer A. This trend is generally followed for the other silencers investigated here, although for silencer D the lower open area causes the scattering from the silencer inlet to play more of a role at higher frequencies. It is, however, encouraging to note that the two methods generally deliver similar values for the IL. This observation has also been confirmed in predictions for other silencers (not shown here) and so it appears reasonable to conclude that one may neglect the influence of the chamfer when predicting silencer performance in the absence of mean flow. This confers a significant advantage because the degrees of freedom required drop significantly; for example, for silencer A they drop from 9,446 to 200.

When a mean flow of Mach number of $M_{5}=0.2$ is added, agreement between the two methods is seen to be comparable to that observed without flow. Therefore, one may conclude that the discrepancy between the two methods seen in Fig. 16 is caused by the presence of the chamfer rather than the way in which the continuity conditions have been enforced. Thus, the use of an abrupt change in the mean flow velocity over an area discontinuity appears not to have any significant affect on the silencer predictions, at least within experimental uncertainty. Therefore, the results presented here show that it is feasible to approximate a bull nose fairing as a flat plate and to permit a jump in mean flow velocity over the silencer entry/exit planes. Any discrepancies caused by this assumption are likely to 
appear at higher frequencies and for relatively high mean flow velocities, but they are likely to be of the same order as those errors observed in experimental measurements. Therefore, in view of the enormous saving in computational expenditure, it appears appropriate in the future to use the point collocation approach of Kirby $[3,17]$ modified to include mean flow. This permits the inclusion of mean flow in an iterative design procedure, which must form an essential part of any silencer design procedure. This is because the number of variables that influence silencer performance is seen here to be considerable. Moreover, the appropriate design of a dissipative silencer will be strongly influenced by the sound power characteristics of the sound source, as well as the size of the duct into which the silencer will be placed. Therefore, it is difficult to use the model generated here to report general guidelines regarding the appropriate design of a splitter silencer in the presence of flow. Accordingly, the purpose of this article is to present and validate a theoretical methodology for use in bespoke design applications and here it is shown that one may use the point collocation method to do this. This simplified approach has significant advantages for larger and more complex silencers such as the three dimensional silencers studied by Kirby et al. [32].

\section{CONCLUSIONS}

The acoustic performance of a dissipative splitter silencer has been studied here using both experimental and theoretical techniques. The theoretical model permits the inclusion of chamfers on the silencer fairings and IL predictions are presented both with and without

mean flow. In the absence of mean flow the theoretical model is shown to be capable of capturing silencer performance over a wide frequency range and for an upper frequency limit of $8 \mathrm{kHz}$. However, for those silencers with a percentage open area below about $30-35 \%$ the theoretical model tends to over predict the measured silencer performance at higher 
frequencies and some uncertainty still persists regarding the reason for these discrepancies. The measurement of silencer performance with mean flow was found to be difficult because of the desire to maintain an incident plane wave. This meant that significant problems were encountered with the signal to noise ratio at higher mean flow velocities. Accordingly, the mean flow measurements were restricted to relatively modest face velocities, although it was still possible to see a lowering of IL at low frequencies. The theoretical model was then used to investigate higher mean flow velocities and the general trends seen in the experiments were also observed in the theoretical predictions. That is, when a mean gas flow is in the direction of sound propagation, silencer performance is seen to drop at low frequencies, whereas at higher frequencies the effect of mean flow is less significant. At low frequencies the opposite is true when the direction of the mean flow is reversed. At higher frequencies the effect of mean flow on silencer IL is less significant and so it appears to be justifiable to neglect mean flow at higher frequencies. This offers significant savings in computational expenditure for the theoretical model, as well as the possibility of redesigning experimental methodologies to focus only on low frequency measurements when mean flow is present.

It is demonstrated here that it is possible to approximate the effects of mean flow on silencer IL by using the point collocation model of Kirby $[3,17]$ suitably modified to include mean flow [18]. This involves removing the bull nose fairing and replacing it with a flat plate so that continuity conditions are enforced over a step change in the mean flow profile. It is concluded that the errors incurred by enforcing continuity conditions over a step velocity change are negligible and that influence of mean flow on silencer performance is dictated by the velocity in the central section of the silencer. Accordingly, the rather onerous hybrid method reported here can be replaced with a relatively straightforward point collocation approach, which significantly lowers computational demands when studying the effects of 
mean flow. This is important in delivering fast and effective commercial design facilities for applications where mean flow velocities may be significant, such as gas turbine exhaust systems. It also delivers a method viable for studying the effects of flow on more complex silencer designs that require a three dimensional approach, see for example the designs studied by Kirby et al. [32].

\section{ACKNOWLEDGEMENTS}

The authors would like to thank the UK Technology Strategy Board (TSB), through the Knowledge Transfer Programme (KTP), for their support of the work reported in this article. The authors would also like to thank CAICE Acoustic Air Movement Ltd. and AAF Ltd. for granting access to their silencer performance measurements, and to AAF Ltd for providing the facility for measuring the material properties. 
Finite element matrices:

$$
\begin{aligned}
& \mathbf{K}_{2}=\int_{\mathrm{R}_{2}}\left(\nabla \mathrm{W}^{\mathrm{T}} \cdot \mathrm{T}_{2} \nabla \mathrm{W}+2 \mathrm{i} k_{0} \mathrm{~W}^{\mathrm{T}} \mathbf{M}_{2} \nabla \mathrm{W}-k_{0}^{2} \mathrm{~W}^{\mathrm{T}} \mathrm{W}\right) d \mathrm{R} \\
& \mathbf{K}_{3}=\beta \int_{\mathrm{R}_{3}}\left(\nabla \mathrm{W}^{\mathrm{T}} \cdot \nabla \mathrm{W}+\Gamma^{2} \mathrm{~W}^{\mathrm{T}} \mathrm{W}\right) d \mathrm{R} \\
& \mathbf{V}_{2}=\frac{\mathrm{i} k_{0}}{\zeta} \int_{\Gamma_{\mathrm{p} 2}}\left(\mathrm{~W}^{\mathrm{T}} \mathrm{W}-2 \mathrm{iM}_{5} \mathrm{~W}^{\mathrm{T}} \frac{\partial \mathrm{W}}{\partial x}-\frac{\mathrm{M}_{5}^{2}}{k_{0}^{2}} \frac{\partial \mathrm{W}^{\mathrm{T}}}{\partial x} \frac{\partial \mathrm{W}}{\partial x}\right) d \Gamma \\
& \mathbf{V}_{3}=\frac{\mathrm{i} k_{0}}{\zeta} \int_{\Gamma_{\mathrm{p} 3}} \mathrm{~W}^{\mathrm{T}} \mathrm{W} d \Gamma \\
& \mathbf{Q}_{i, r}=\mathrm{i} k_{0}\left[1-\mathrm{M}_{1}^{2}\right] \gamma_{i, r}^{m} \int_{\Gamma_{\mathrm{A}}} \Phi^{m} \mathrm{~W} d \Gamma, \quad m=0,1,2, \ldots . n_{1} . \\
& \mathbf{R}_{i, r}=\mathrm{i} k_{0} \lambda_{i, r}^{m}\left[\left[1-\mathrm{M}_{5}^{2}\right] \int_{\Gamma_{\mathrm{B} 2}} \Psi_{i, r}^{m} \mathrm{~W} d \Gamma+\beta \int_{\Gamma_{\mathrm{B} 3}} \Psi_{i, r}^{m} \mathrm{~W} d \Gamma\right], m=0,1,2, \ldots . n_{\mathrm{BC} .}
\end{aligned}
$$

$$
\mathbf{M}_{1 i, r}=\mathrm{i} k_{0}\left(1-\mathrm{M}_{1}^{2}\right) \gamma_{r}^{m} \int_{\Gamma_{\mathrm{A}}} \Phi^{m} \Phi^{n} d \Gamma, \quad m=0,1,2, \ldots n_{1}, n=0,1,2, \ldots n_{1}
$$$$
\mathbf{M}_{2 i, r}=\mathrm{i} k_{0} \lambda_{i}^{m}\left[\left[1-\mathrm{M}_{5}^{2}\right] \int_{\Gamma_{\mathrm{B} 2}} \Psi_{i}^{m} \Psi_{i, r}^{m} d \Gamma+\beta \int_{\Gamma_{\mathrm{B} 3}} \Psi_{i}^{m} \Psi_{i, r}^{m} d \Gamma\right]
$$$$
m=0,1,2, \ldots . n_{\mathrm{BC}}, n=0,1,2, \ldots . n_{\mathrm{BC}}
$$

$$
\mathbf{K}_{7}=\int_{\mathrm{R}_{7}}\left(\nabla \mathrm{W}^{\mathrm{T}} \cdot \mathbf{T}_{7} \nabla \mathrm{W}+2 \mathrm{i} k_{0} \mathrm{~W}^{\mathrm{T}} \mathbf{M}_{7} \nabla \mathrm{W}-k_{0}^{2} \mathrm{~W}^{\mathrm{T}} \mathrm{W}\right) d \mathrm{R}
$$

$$
\mathbf{K}_{8}=\beta \int_{\mathrm{R}_{8}}\left(\nabla \mathrm{W}^{\mathrm{T}} \cdot \nabla \mathrm{W}+\Gamma^{2} \mathrm{~W}^{\mathrm{T}} \mathrm{W}\right) d \mathrm{R}
$$




$$
\begin{aligned}
& \mathbf{V}_{7}=\frac{\mathrm{i} k_{0}}{\zeta} \int_{\Gamma_{\mathrm{p} 7}}\left(\mathrm{~W}^{\mathrm{T}} \mathrm{W}-2 \mathrm{iM}_{5} \mathrm{~W}^{\mathrm{T}} \frac{\partial \mathrm{W}}{\partial x}-\frac{\mathrm{M}_{5}^{2}}{k_{0}^{2}} \frac{\partial \mathrm{W}^{\mathrm{T}}}{\partial x} \frac{\partial \mathrm{W}}{\partial x}\right) d \Gamma \\
& \mathbf{V}_{8}=\frac{\mathrm{i} k_{0}}{\zeta} \int_{\Gamma_{\mathrm{p} 8}} \mathrm{~W}^{\mathrm{T}} \mathrm{W} d \Gamma \\
& \mathbf{Y}_{i, r}=\mathrm{i} k_{0}\left[1-\mathrm{M}_{10}^{2}\right] \gamma_{i, r}^{m} \int_{\Gamma_{\mathrm{D}}} \Phi^{m} \mathrm{~W} d \Gamma, \quad m=0,1,2, \ldots . n_{1} . \\
& \mathbf{X}_{i, r}=\mathrm{i} k_{0} \lambda_{i, r}^{m}\left[\left[1-\mathrm{M}_{5}^{2}\right] \int_{\Gamma_{\mathrm{C} 2}} \Psi_{i, r}^{m} \mathrm{~W} d \Gamma+\beta \int_{\Gamma_{\mathrm{C} 3}} \Psi_{i, r}^{m} \mathrm{~W} d \Gamma\right] m=0,1,2, \ldots . n_{\mathrm{BC}} \\
& \mathbf{M}_{10 i, r}=\mathrm{i} k_{0}\left(1-\mathrm{M}_{10}^{2}\right) \gamma_{r}^{m} \int_{\Gamma_{\mathrm{D}}} \Phi^{m} \Phi^{n} d \Gamma, \quad m=0,1,2, \ldots . n_{1}, n=0,1,2, \ldots n_{1} \\
& \mathbf{M}_{7 i, r}=\mathrm{i} k_{0} \lambda_{i}^{m}\left[\left[1-\mathrm{M}_{5}^{2}\right] \int_{\Gamma_{\mathrm{C} 2}} \Psi_{i}^{m} \Psi_{i, r}^{m} d \Gamma+\beta \int_{\Gamma_{\mathrm{C} 3}} \Psi_{i}^{m} \Psi_{i, r}^{m} d \Gamma\right] \\
& m=0,1,2, \ldots . n_{\mathrm{BC}}, n=0,1,2, \ldots . n_{\mathrm{BC}} .
\end{aligned}
$$

The matrices $\mathbf{E}_{i, r}$ are diagonal matrices in which each element is given by $\mathrm{e}^{\mp \mathrm{i} k_{0} \lambda_{i, r}^{m} L_{\mathrm{BC}}}(m=$ $\left.0,1, \ldots, n_{\mathrm{BC}}\right)$, with the - sign being used for the incident (i) mode and the + sign for the reflected $(r)$ mode 


\section{REFERENCES}

1. B. Nennig, M. Ben Tahar, E. Perrey-Debain, A displacement-pressure finite element formulation for analyzing the sound transmission in ducted shear flows with finite poroelastic lining, Journal of the Acoustical Society of America 130 (2011) 42-51.

2. B. Nennig, E. Perrey-Debain, M. Ben Tahar, A mode matching method for modeling dissipative silencers lined with poroelastic materials and containing mean flow, Journal of the Acoustical Society of America 128 (2010) 3308-3320.

3. R. Kirby, A comparison between analytic and numerical methods for modelling automotive dissipative silencers with mean flow, Journal of Sound and Vibration 325 (2009) 565-582.

4. R. Kirby, F.D. Denia, Analytic mode matching for a circular dissipative silencer containing mean flow and a perforated pipe, Journal of the Acoustical Society of America $122(2007) 3471-3482$.

5. R. Kirby, Transmission loss predictions for dissipative silencers of arbitrary cross section in the presence of mean flow, Journal of the Acoustical Society of America 114 (2003) 200-209.

6. K.S. Peat, K.L. Rathi, A finite element analysis of the convected acoustic wave motion in dissipative silencers, Journal of Sound and Vibration 184 (1995) 529-545.

7. S.-H. Ko, Sound attenuation in acoustically lined circular ducts in the presence of uniform flow and shear flow, Journal of Sound and Vibration 22 (1972) 193-210.

8. S.-H. Ko, Theoretical analyses of sound attenuation in acoustically lined flow ducts separated by porous splitters (rectangular, annular and circular ducts), Journal of Sound and Vibration 39 (1975) 471-487. 
9. D.A. Bies, C.H. Hansen and G.E. Bridges, Sound attenuation in rectangular and circular cross-section ducts with flow and bulk-reacting liner, Journal of Sound and Vibration 146 (1991) 47-80.

10. A. Cummings, N. Sormaz, Acoustic attenuation in dissipative splitter silencers containing mean fluid flow, Journal of Sound and Vibration 168 (1993) 209-227.

11. S.K. Kakoty, V.K. Roy, Bulk reaction modeling of ducts with and without mean flow, Journal of the Acoustical Society of America 112 (2002) 75-112.

12. P. Brandstätt, W. Frommhold and M.J. Fisher, Program for the computation of absorptive silencers in straight ducts, Applied Acoustics 43 (1994) 19-38.

13. R.J. Astley, W. Eversman, A finite element formulation of the eigenvalue problem in lined ducts with flow, Journal of Sound and Vibration 65 (1979) 61-74.

14. R.J. Astley, A. Cummings, A finite element scheme for attenuation in ducts lined with porous material, Journal of Sound and Vibration 116 (1987) 239-263.

15. R. Kirby, J.B. Lawrie, A point collocation approach to modelling large dissipative silencers, Journal of Sound and Vibration 286 (2005) 313-339.

16. J.B. Lawrie, R. Kirby, Mode matching without root finding: Application to a dissipative silencer, Journal of the Acoustical Society of America 119 (2006) 2050-2061.

17. R. Kirby, The influence of baffle fairings on the acoustic performance of rectangular splitter silencers, Journal of the Acoustical Society of America, 118 (2005) 2302-2312.

18. R. Kirby, Modeling sound propagation in acoustic waveguides using a hybrid numerical method, Journal of the Acoustical Society of America 124 (2008) 1930-1940.

19. W. Eversman, Mapped infinite wave envelope elements of acoustic radiation in a uniformly moving medium, Journal of Sound and Vibration 224 (1999) 665-687.

20. A.D. Pierce, Wave equation for sound in fluids with unsteady inhomogeneous flow, Journal of the Acoustical Society of America 87 (1990) 2292-2299. 
21. F.P. Mechel, Numerical results to the theory of baffle-type silencers, Acustica 72 (1990) $7-20$.

22. C.L. Morfey, Sound transmission and generation in ducts with flow, Journal of Sound and Vibration 14 (1971) 37-55.

23. European Standard EN ISO 7235, "Measurement procedures for ducted silencers insertion loss, flow noise and total pressure loss," (2003).

24. ASTM E477, "Standard Test Method for Measuring Acoustical and Airflow Performance of Duct Liner Materials and Prefabricated Silencers,” DOI. 10.1520/E0477-06A.

25. European Standard EN ISO 10534-2:2001, "Determination of sound absorption coefficient and impedance in impedance tubes. Transfer function method." (2001).

26. P.T. Williams, R. Kirby, C. Malecki, J. Hill, Measurement of the bulk acoustic properties of fibrous materials at high temperatures, Applied Acoustics 77 (2014) 29-36.

27. European Standard EN ISO 29053:1993, “Acoustics: Materials for acoustical applications. Determination of airflow resistance.” (1993).

28. R. Kirby and A. Cummings, Prediction of the bulk acoustic properties of fibrous materials at low frequencies, Applied Acoustics 56 (1999) 101-125.

29. R. Kirby and A. Cummings, The impedance of perforated plates subjected to grazing mean flow and backed by porous media, Journal of Sound and Vibration 217 (1998) 619636.

30. F.D. Denia, A. Selamet, F.J. Fuenmayor and R. Kirby, Acoustic attenuation performance of perforated dissipative mufflers with empty inlet/outlet extensions, Journal of Sound and Vibration 302 (2007) 1000-1017.

31. J. Roland, Evaluation of the errors in the measurements of silencer characteristics, Journal of Sound and Vibration 75 (1981) 549-558. 
32. R. Kirby, P.T. Williams, J. Hill, A three dimensional investigation into the acoustic performance of dissipative splitter silencers, Journal of the Acoustical Society of America 135 (2014) 2727-2737. 


\section{Figure Captions}

Figure 1. Geometry of splitter silencer.

Figure 2. Geometry of bull nose fairings with chamfer.

Figure 3. Geometry of experimental test rig.

Figure 4. Measured and predicted IL for silencer A in the absence of mean flow. prediction; $\boldsymbol{\Lambda}$, measurement.

Figure 5. Measured and predicted IL for silencer B in the absence of mean flow. prediction; $\boldsymbol{\Delta}$, measurement.

Figure 6. Measured and predicted IL for silencer $\mathrm{C}$ in the absence of mean flow. prediction; $\boldsymbol{\Delta}$, measurement.

Figure 7. Measured and predicted IL for silencer D in the absence of mean flow. prediction; $\boldsymbol{\Delta}$, measurement.

Figure 8. Measured and predicted IL for silencer A with a mean flow Mach number of $M_{5}=$ 0.022 in the airway. $\longrightarrow$, prediction; $\boldsymbol{\Delta}$, measurement.

Figure 9. Measured and predicted IL for silencer D with a mean flow Mach number of $M_{5}=$ 0.043 in the airway. $\longrightarrow$, prediction; $\boldsymbol{\Delta}$, measurement. 
Figure 10. IL predictions for Silencer A. $\longrightarrow, M_{5}=0 ;----, M_{5}=0.1 ;-\cdots-$, $\mathrm{M}_{5}=0.2 ;---, \mathrm{M}_{5}=-0.1 ;-\cdots-\cdots, \mathrm{M}_{5}=-0.2$.

Figure 11. IL predictions for Silencer B. $\longrightarrow, M_{5}=0 ;----, M_{5}=0.1 ;-\cdots-$; $\mathrm{M}_{5}=0.2 ;---, \mathrm{M}_{5}=-0.1 ;-\cdot-\cdots, \mathrm{M}_{5}=-0.2$.

Figure 12. IL predictions for Silencer C. $\longrightarrow, M_{5}=0 ;----, M_{5}=0.1 ;-\cdots-$; $\mathrm{M}_{5}=0.2 ;---, \mathrm{M}_{5}=-0.1 ;-\cdot-\cdots, \mathrm{M}_{5}=-0.2$.

Figure 13. IL predictions for Silencer D. $\longrightarrow, M_{5}=0 ;----, M_{5}=0.1 ;-\cdots-$; $\mathrm{M}_{5}=0.2 ;---, \mathrm{M}_{5}=-0.1 ;-\cdot-\cdots, \mathrm{M}_{5}=-0.2$.

Figure 14. Measured and predicted IL in the absence of mean flow.—, point collocation prediction without chamfer; - - - , full hybrid method with chamfer.

Figure 15. Measured and predicted IL with $M_{5}=0.2 . \longrightarrow$, point collocation prediction without chamfer; - - - - , full hybrid method with chamfer. 


\begin{tabular}{|c|c|c|c|c|c|c|}
\hline \multicolumn{7}{|c|}{ Table 1 Test Silencer Geometries } \\
\hline Silencer & $a(\mathrm{~m})$ & $b(\mathrm{~m})$ & $L(\mathrm{~m})$ & $r_{b}$ & $w_{b}$ & $\Delta(\%)$ \\
\hline $\mathrm{A}$ & 0.1 & 0.1 & 1.8 & 0.025 & 0.15 & 50 \\
\hline $\mathrm{B}$ & 0.15 & 0.15 & 0.6 & 0.025 & 0.25 & 50 \\
\hline $\mathrm{C}$ & 0.1 & 0.0625 & 1.2 & 0.025 & 0.15 & 38.5 \\
\hline $\mathrm{D}$ & 0.1 & 0.025 & 0.6 & 0.025 & 0.15 & 20 \\
\hline
\end{tabular}




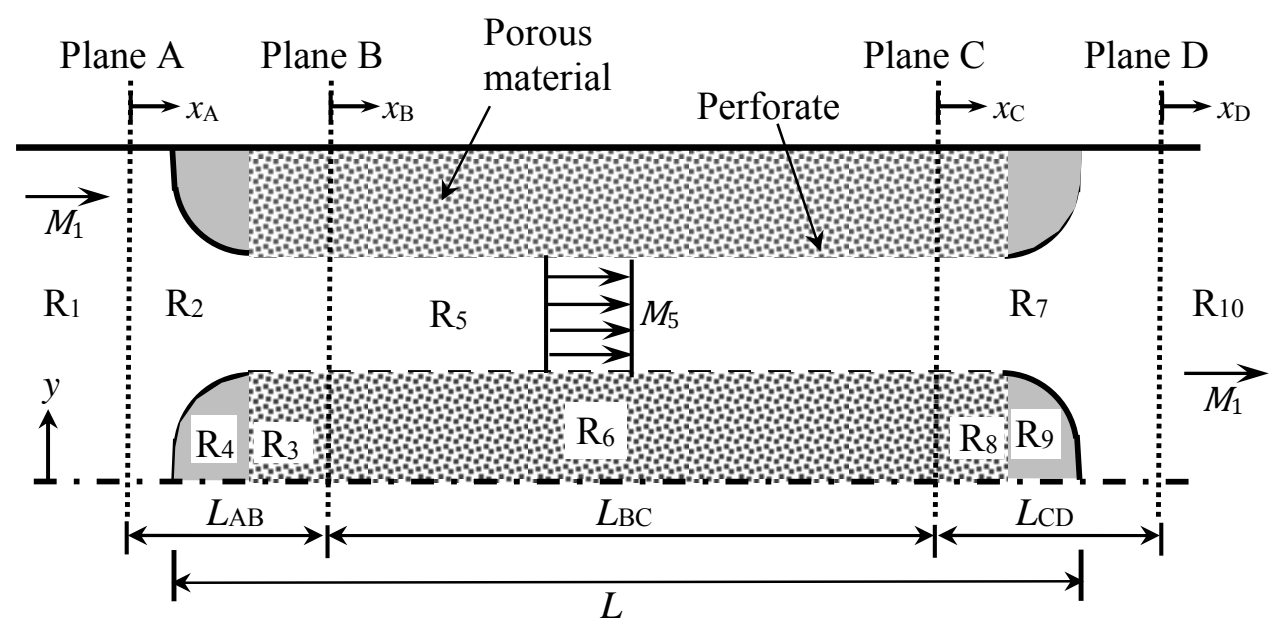

Figure 1. Geometry of splitter silencer. 


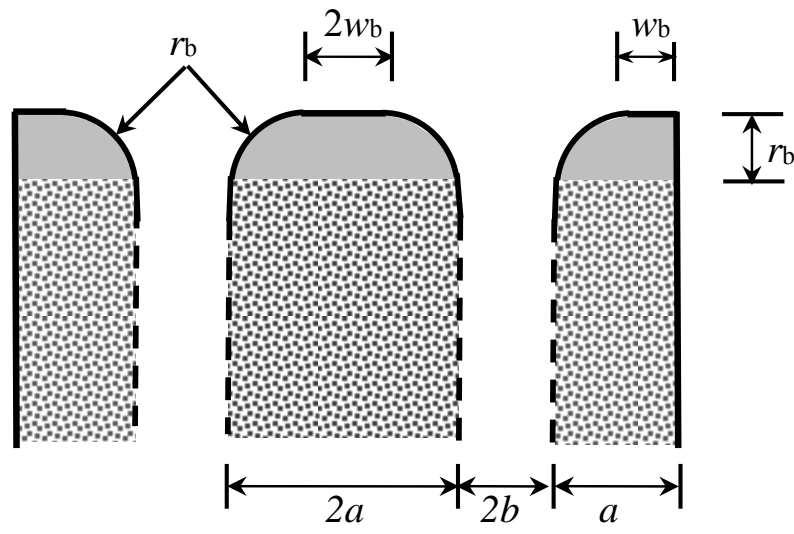

Figure 2. Geometry of bull nose fairings with chamfer. 


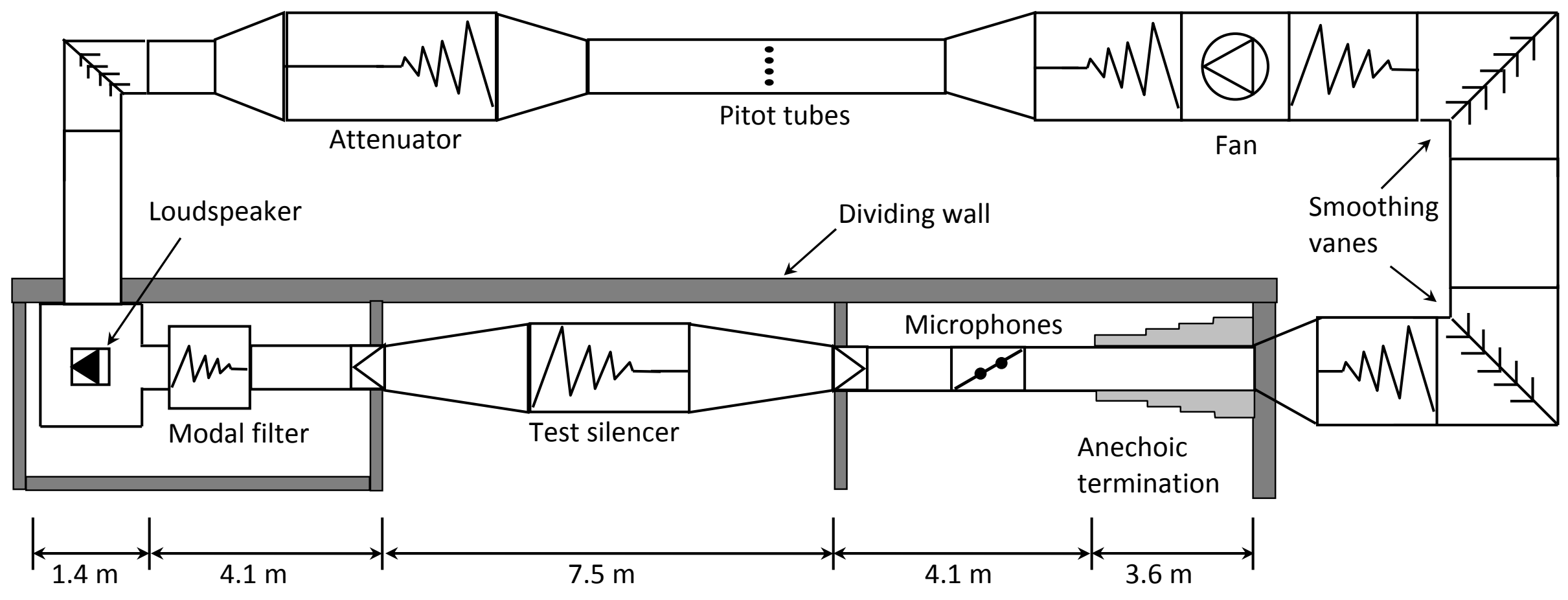

Figure 3. Geometry of experimental test rig. 


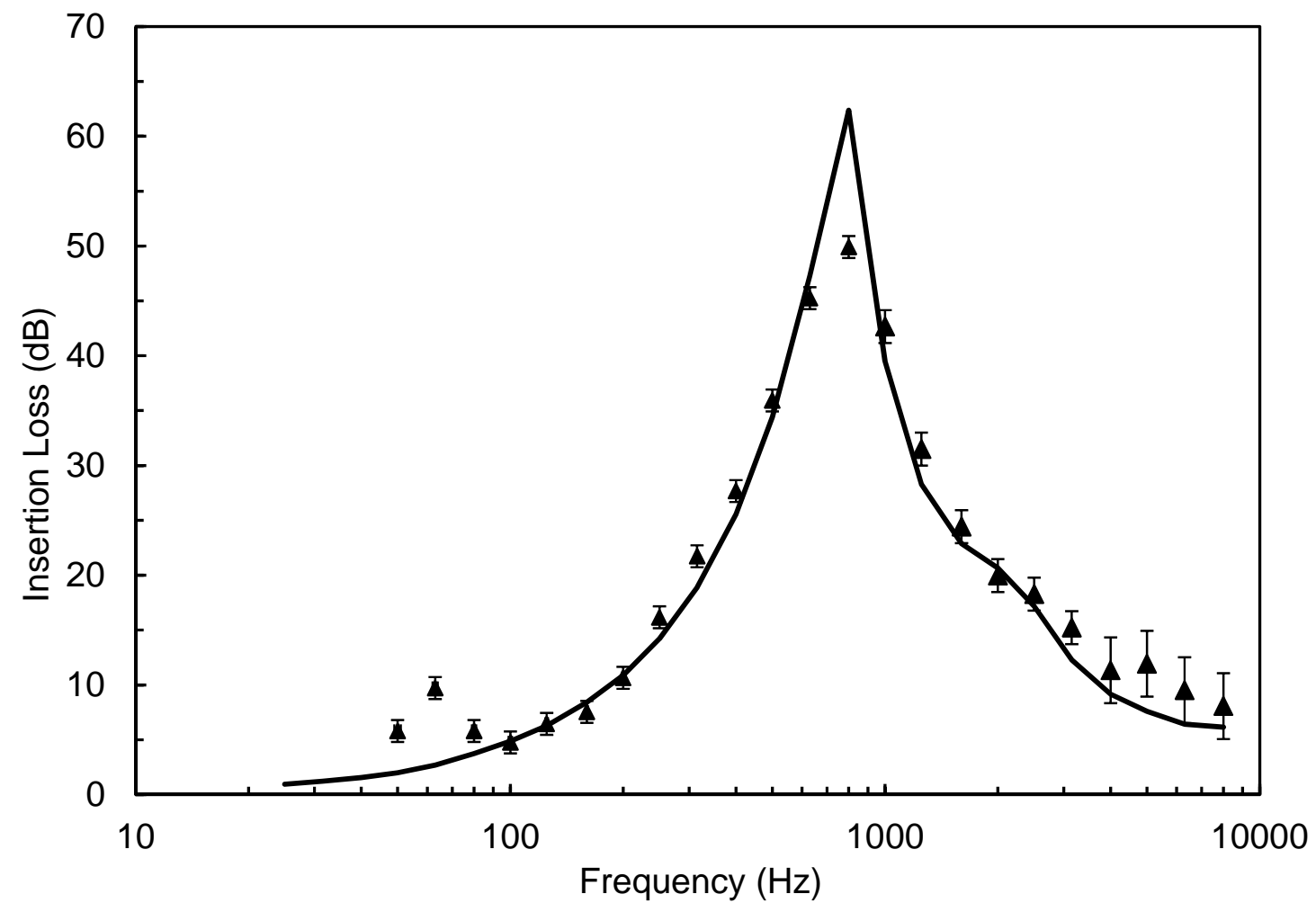

Figure 4. Measured and predicted IL for silencer A in the absence of mean flow.prediction; $\boldsymbol{\Delta}$, measurement. 


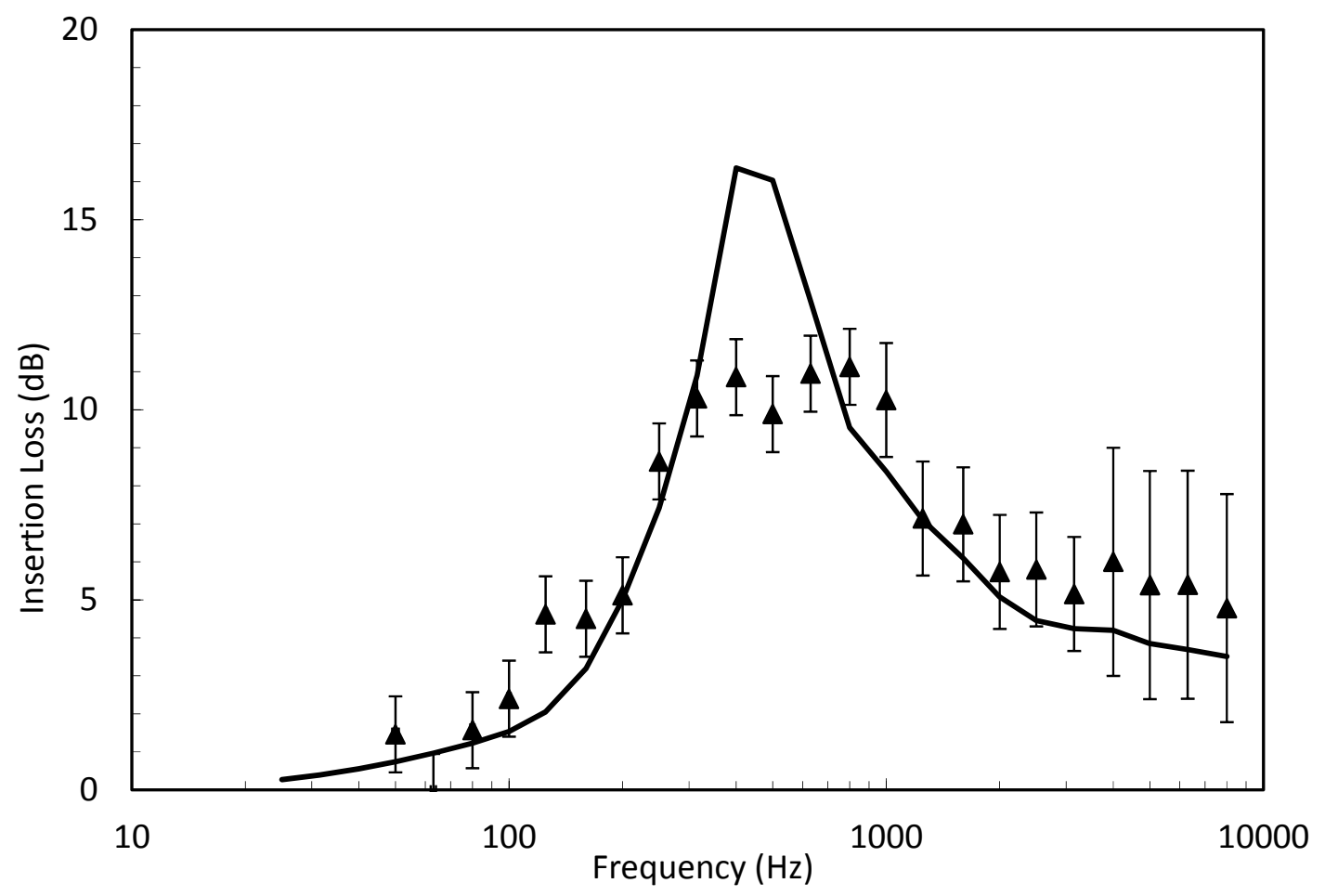

Figure 5. Measured and predicted IL for silencer B in the absence of mean flow. prediction; $\boldsymbol{\Delta}$, measurement. 


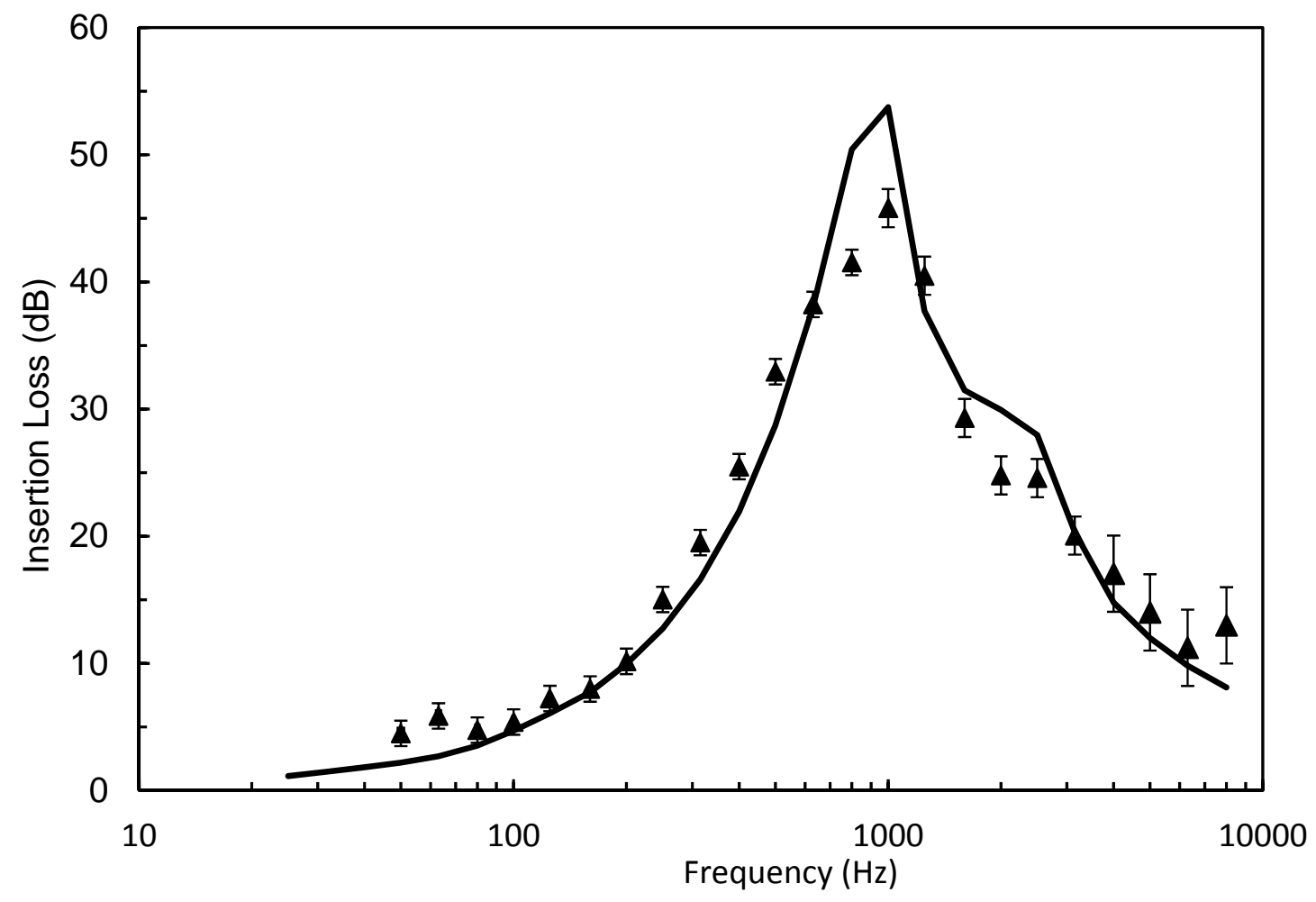

Figure 6. Measured and predicted IL for silencer $\mathrm{C}$ in the absence of mean flow. prediction; $\boldsymbol{\Delta}$, measurement. 


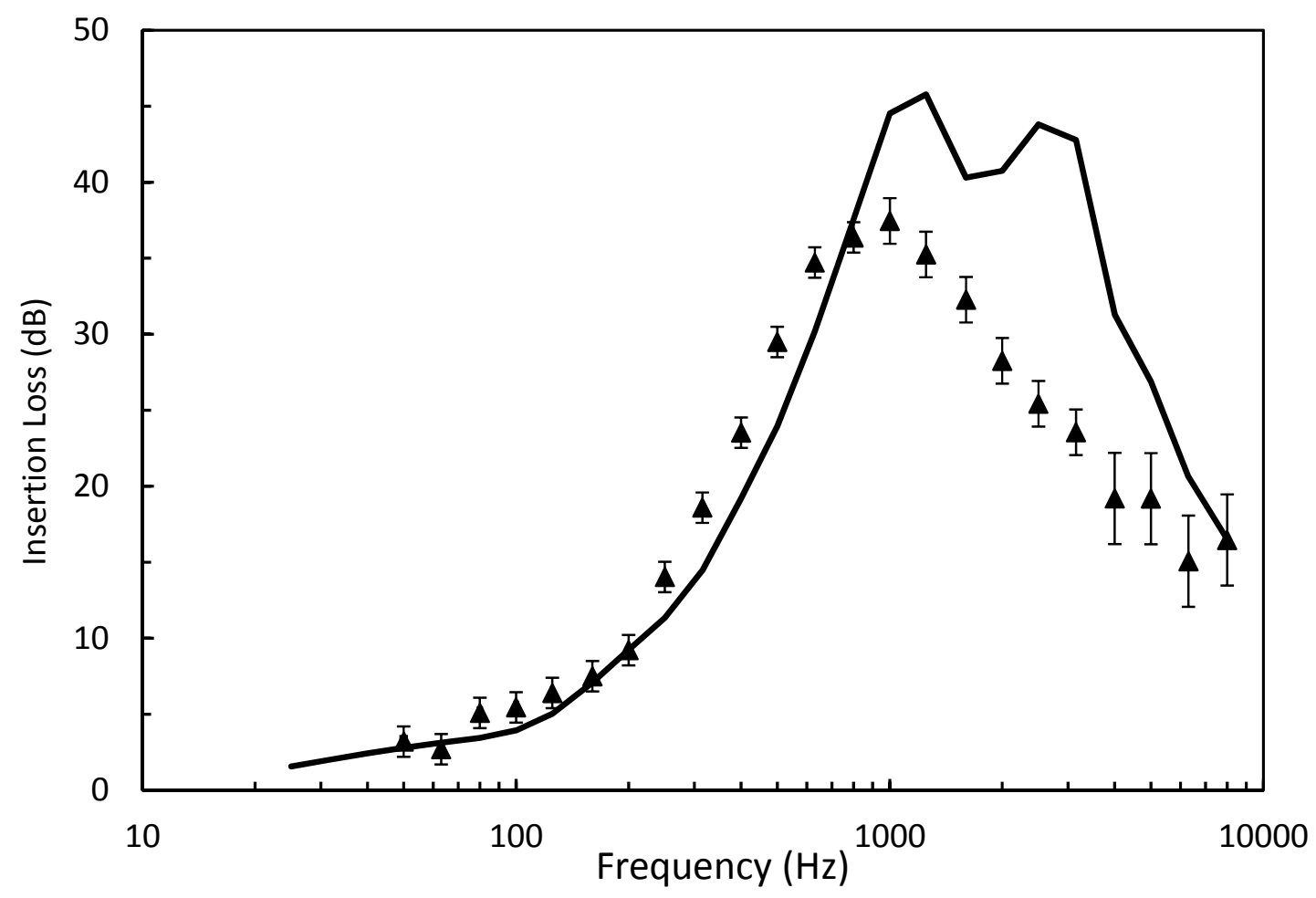

Figure 7. Measured and predicted IL for silencer D in the absence of mean flow. prediction; $\boldsymbol{\Delta}$, measurement. 


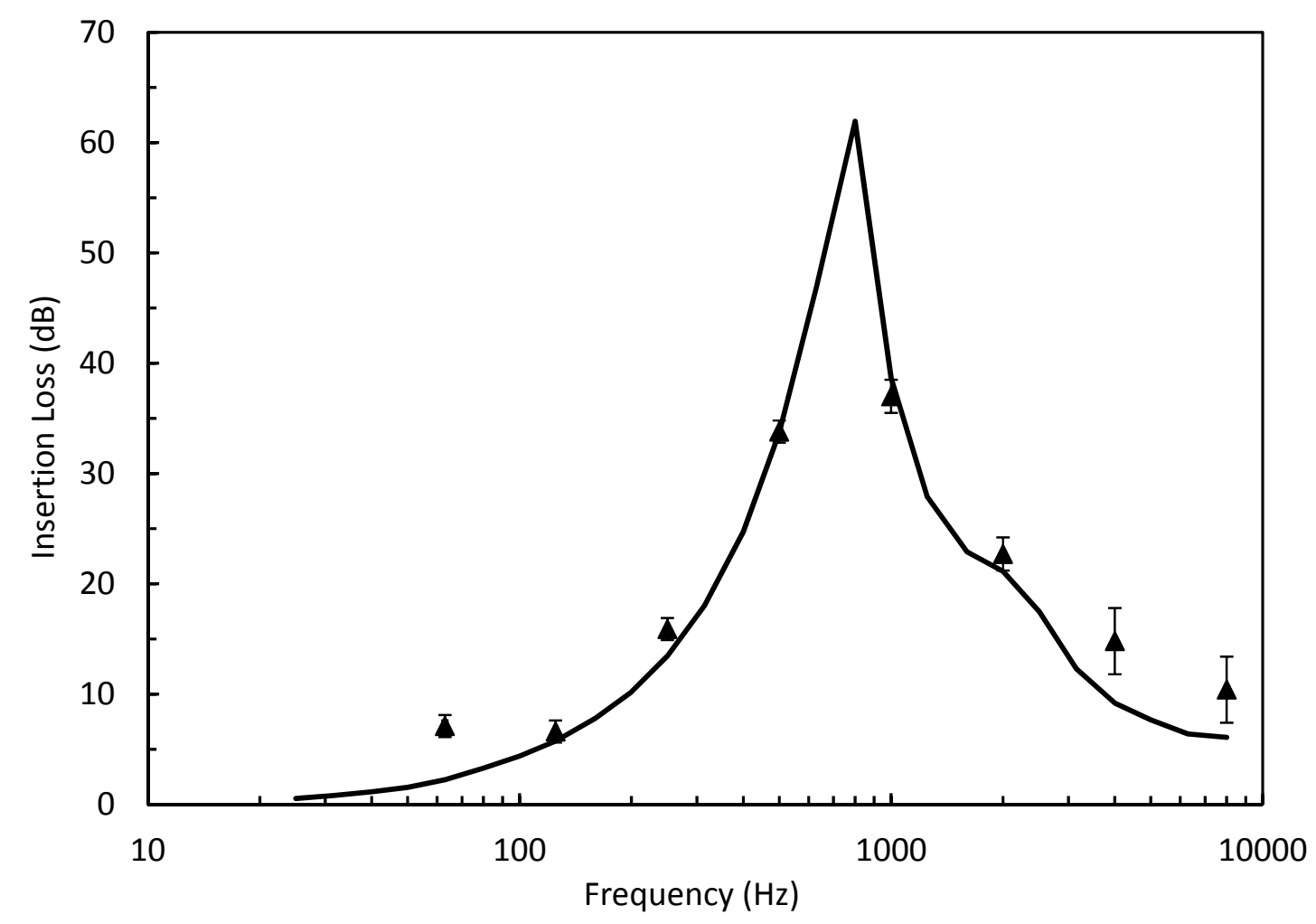

Figure 8. Measured and predicted IL for silencer A with a mean flow Mach number of $M_{5}=$ 0.022 in the airway. $\longrightarrow$, prediction; $\boldsymbol{\Lambda}$, measurement. 


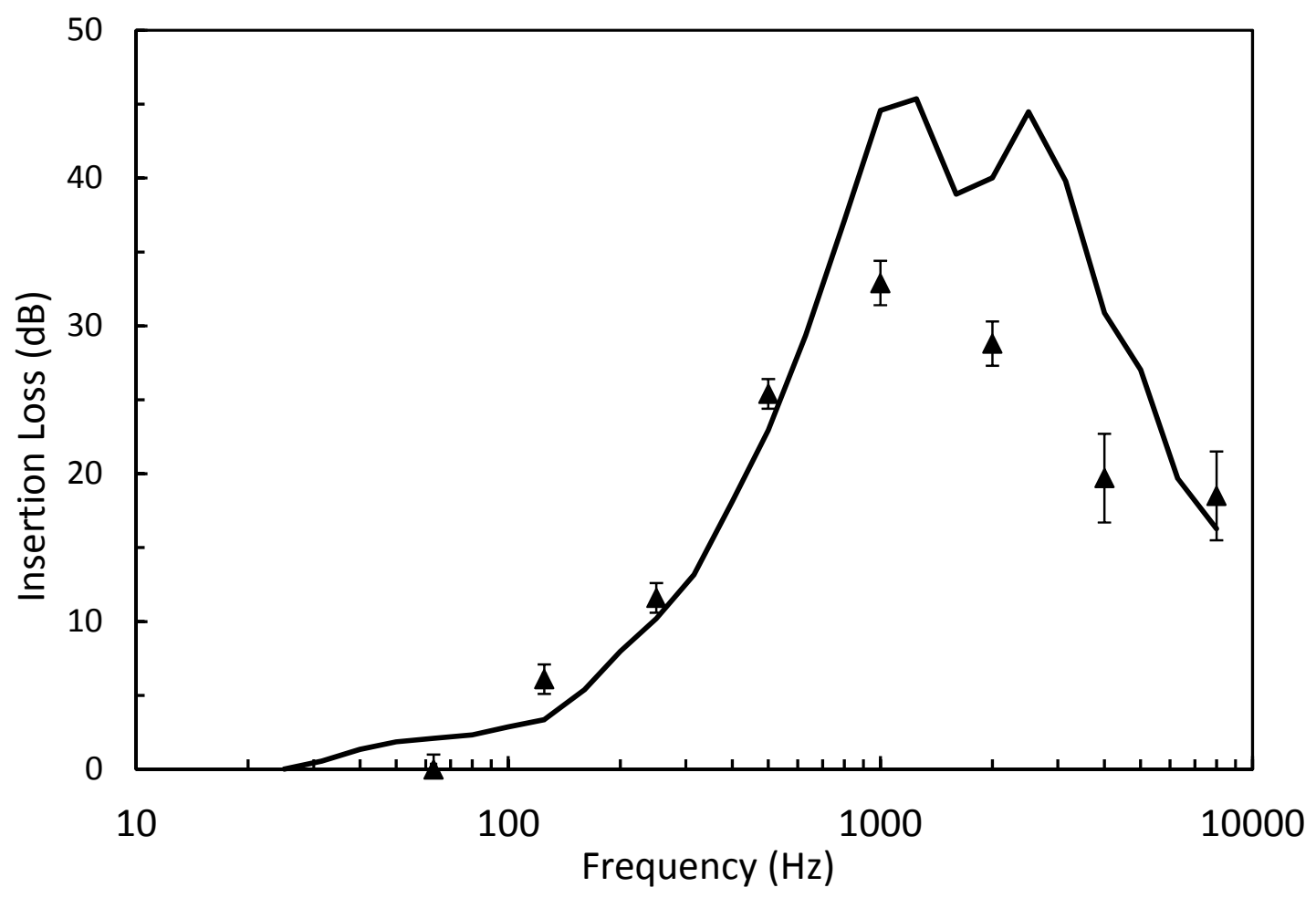

Figure 9. Measured and predicted IL for silencer D with a mean flow Mach number of $M_{5}=$ 0.043 in the airway. $\longrightarrow$, prediction; $\mathbf{\Delta}$, measurement. 


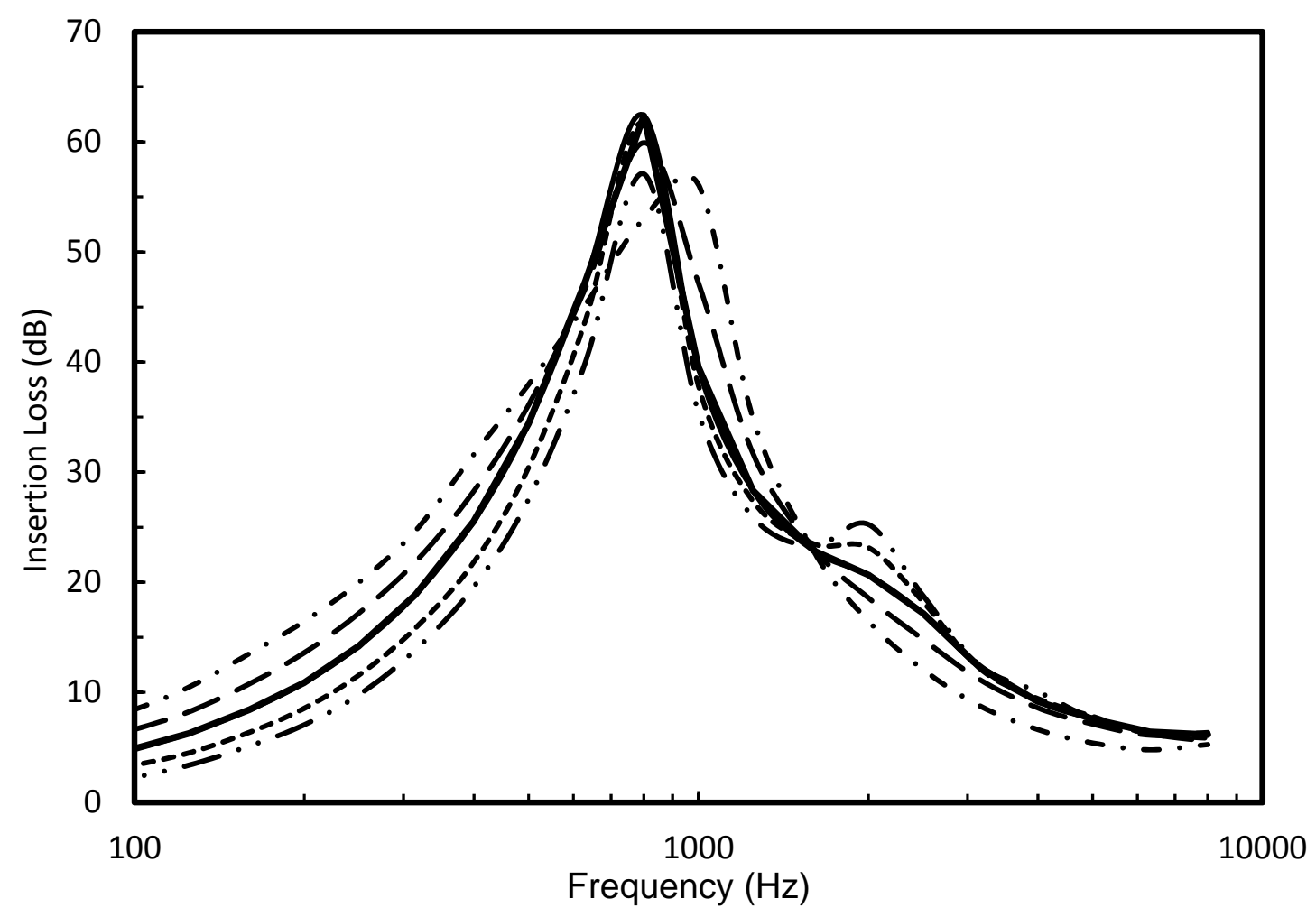

Figure 10. IL predictions for Silencer A. $\longrightarrow, \mathrm{M}_{5}=0 ;----, \mathrm{M}_{5}=0.1 ;-\cdots-\cdot$, $\mathrm{M}_{5}=0.2 ;-\longrightarrow-, \mathrm{M}_{5}=-0.1 ;-\cdot-\cdot, \mathrm{M}_{5}=-0.2$. 


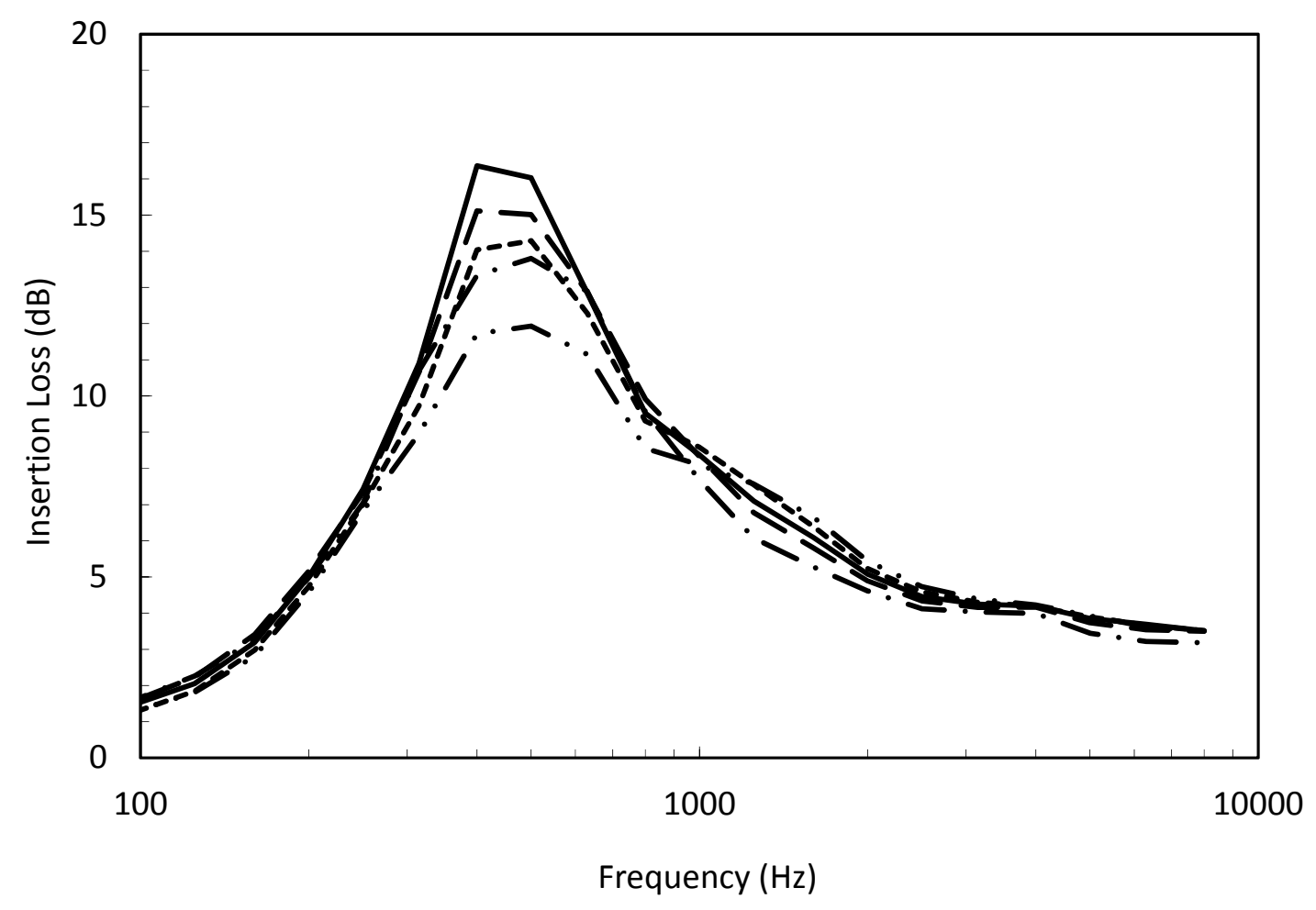

Figure 11. IL predictions for Silencer B. $\longrightarrow, \mathrm{M}_{5}=0 ;----, \mathrm{M}_{5}=0.1 ;-\cdots-\cdot$, $\mathrm{M}_{5}=0.2 ;---, \mathrm{M}_{5}=-0.1 ;-\cdots-\cdots, \mathrm{M}_{5}=-0.2$. 


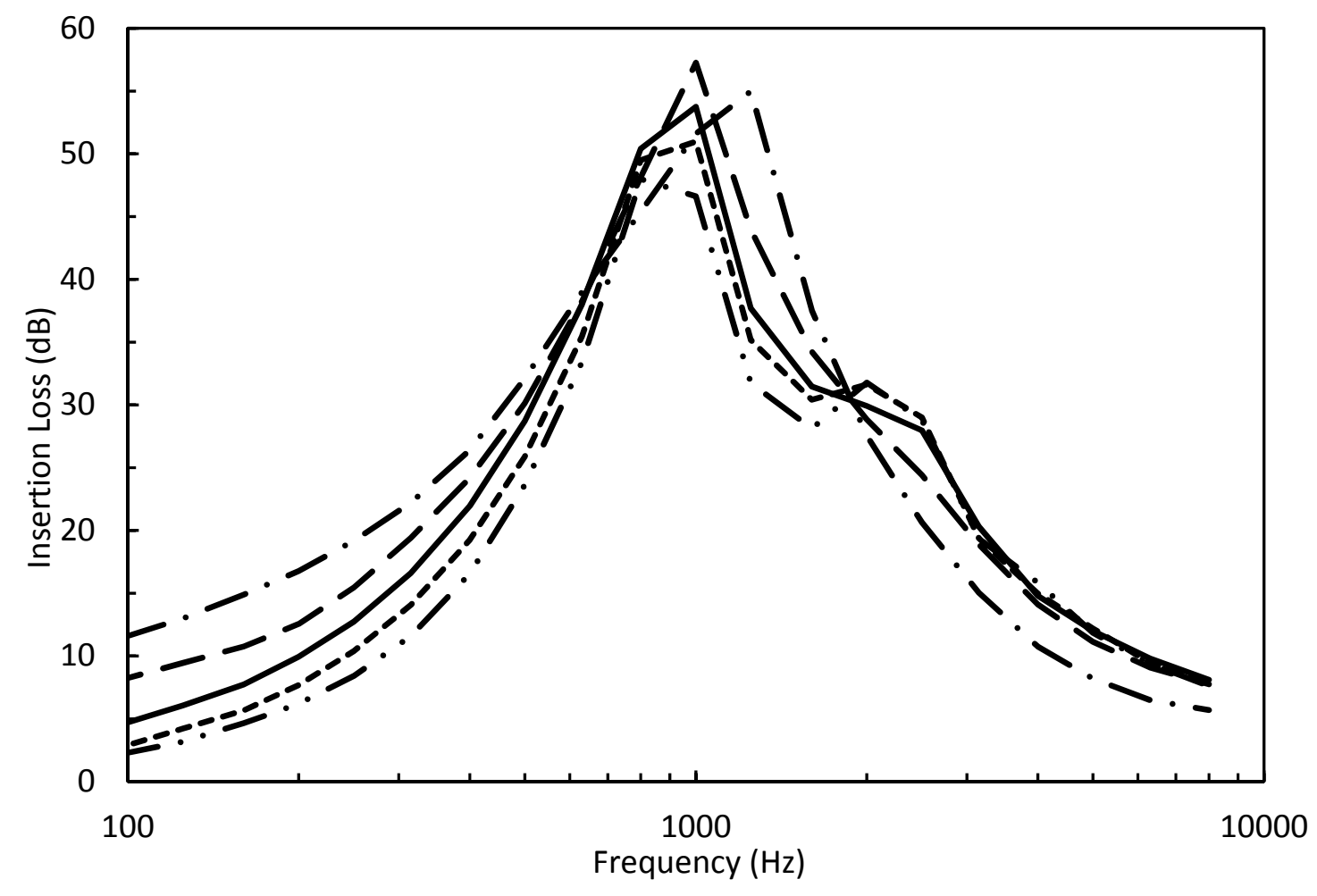

Figure 12. IL predictions for Silencer C. $\longrightarrow, \mathrm{M}_{5}=0 ;----, \mathrm{M}_{5}=0.1 ;-\cdots-\cdot$, $\mathrm{M}_{5}=0.2 ;--\longrightarrow, \mathrm{M}_{5}=-0.1 ;-\cdots-\cdots, \mathrm{M}_{5}=-0.2$. 


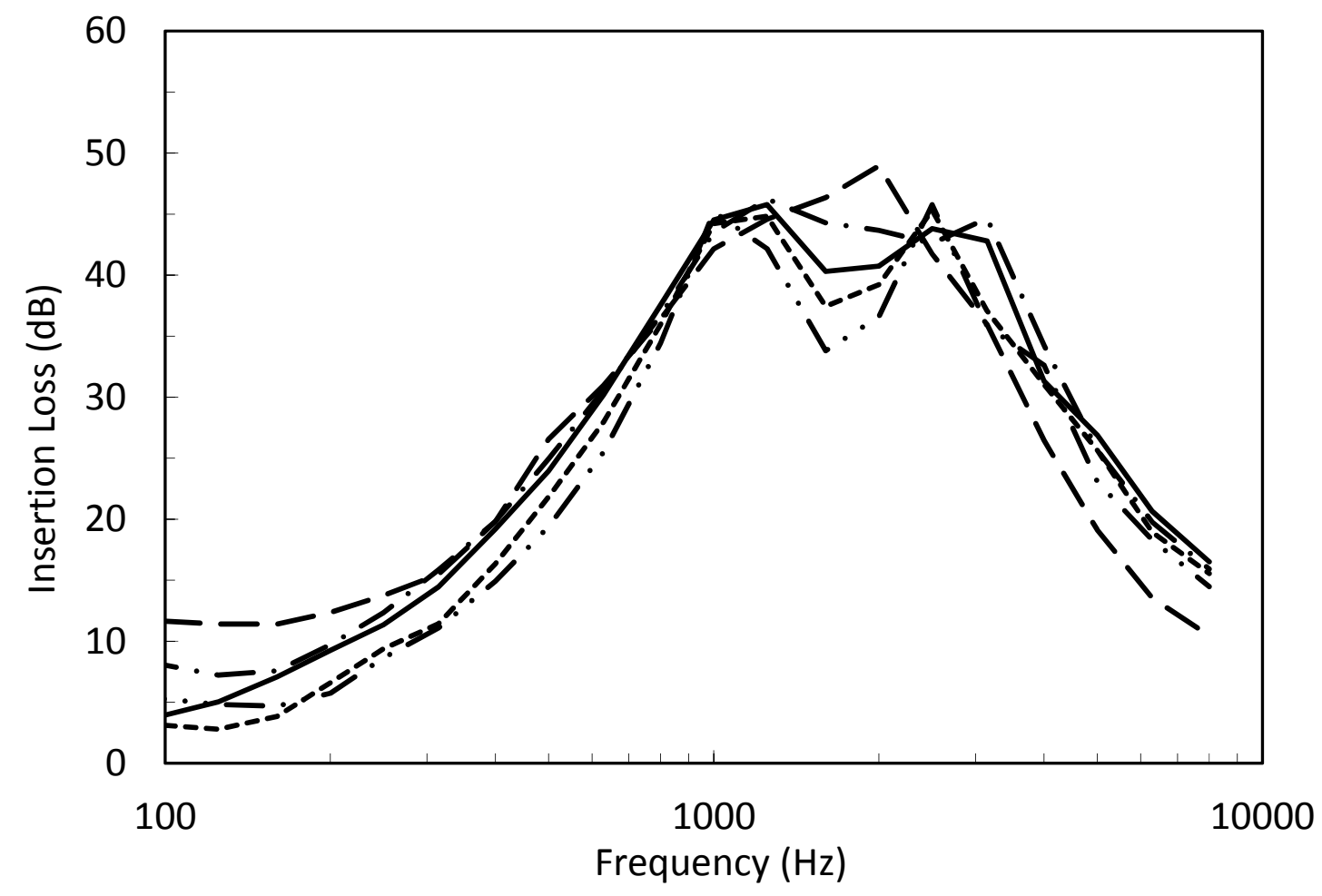

Figure 13. IL predictions for Silencer D. $\longrightarrow, \mathrm{M}_{5}=0 ;----, \mathrm{M}_{5}=0.1 ;-\cdots-\cdot$, $\mathrm{M}_{5}=0.2 ;--\longrightarrow, \mathrm{M}_{5}=-0.1 ;-\cdot-\cdots, \mathrm{M}_{5}=-0.2$. 


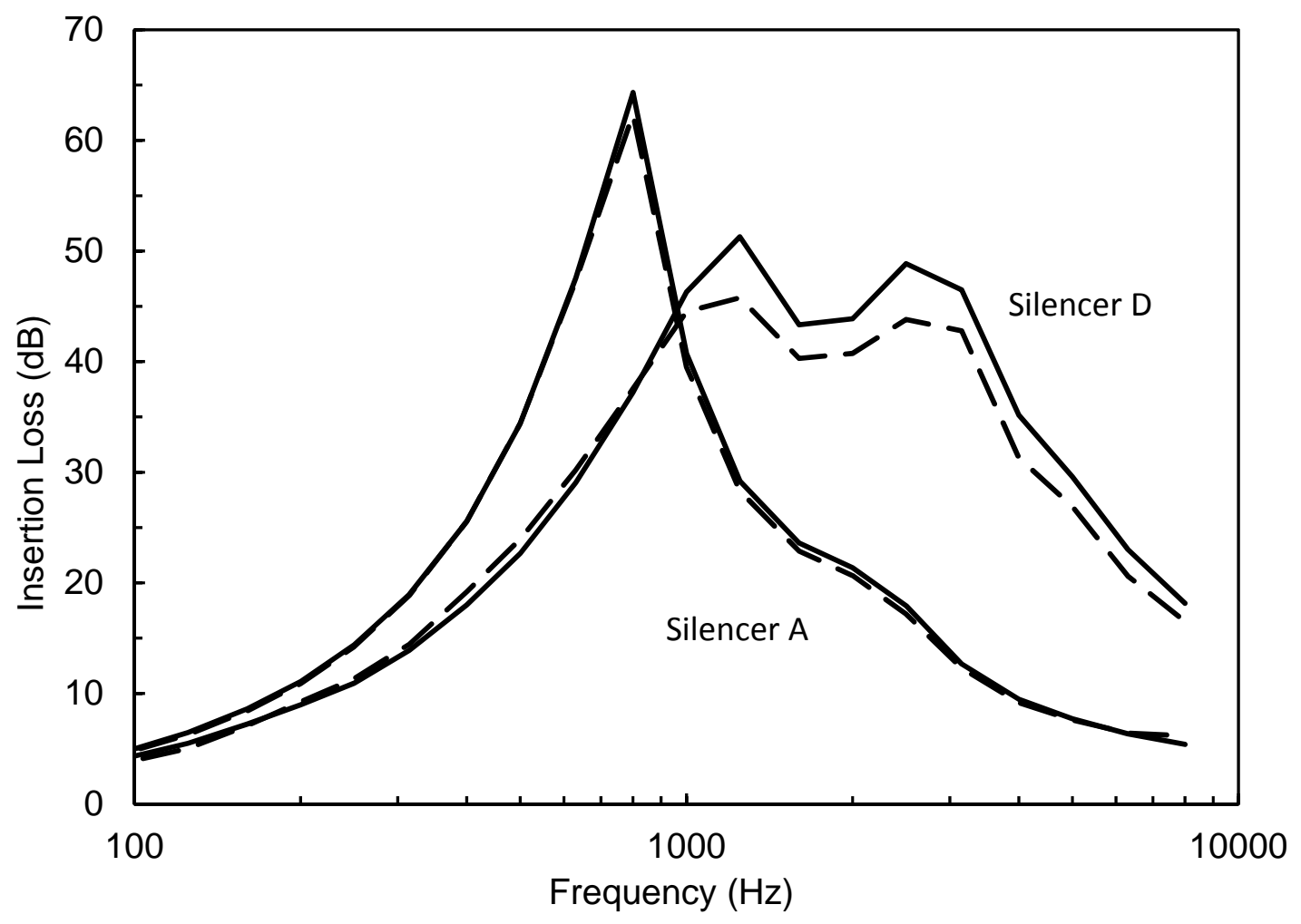

Figure 14. Measured and predicted IL in the absence of mean flow. - , point collocation prediction without chamfer; $-ー-ー$, full hybrid method with chamfer. 


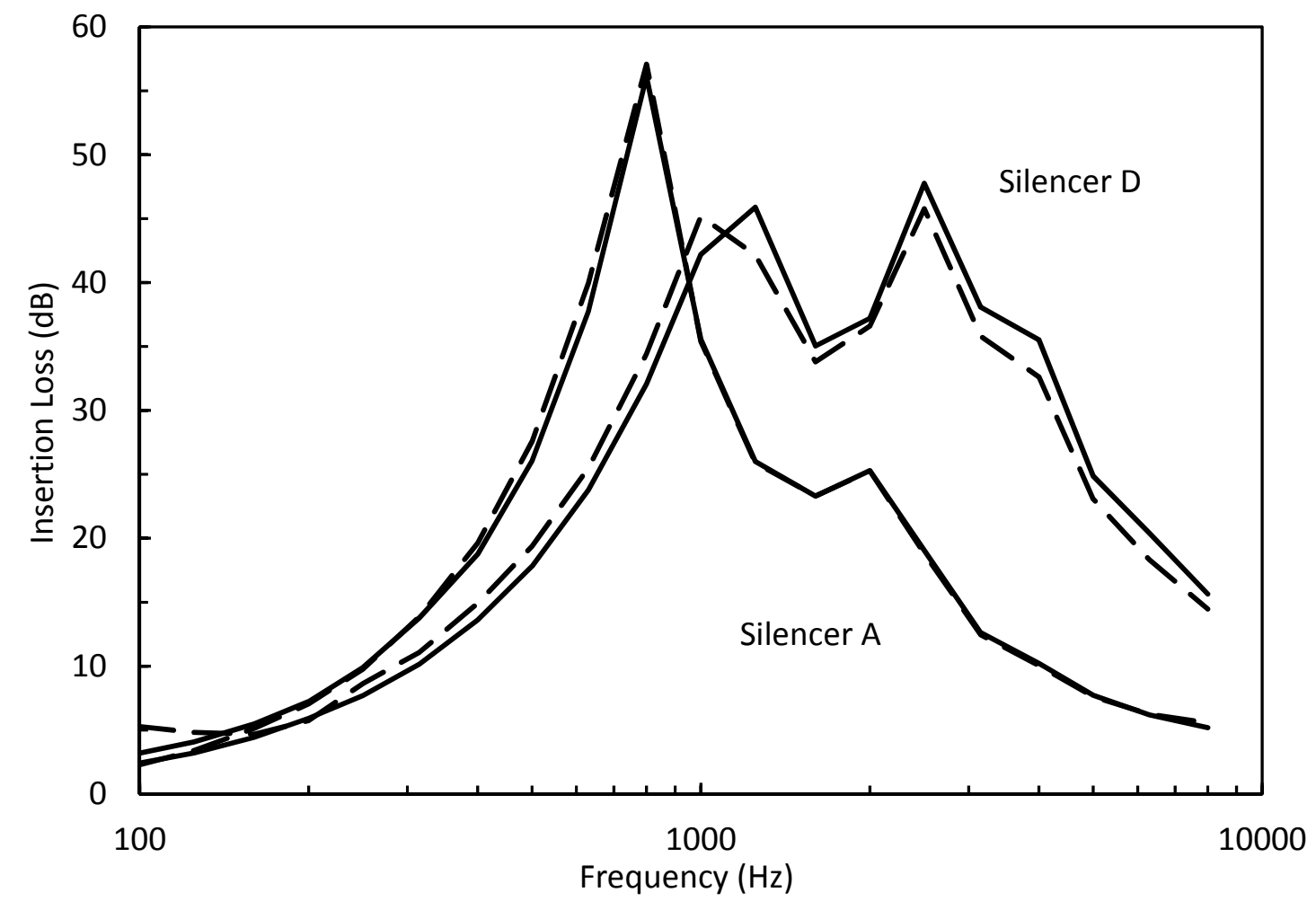

Figure 15. Measured and predicted IL with $\mathrm{M}_{5}=0.2$. $\longrightarrow$, point collocation prediction without chamfer; - - - , full hybrid method with chamfer. 